\title{
Fermi-edge singularity and the functional renormalization group
}

\author{
Fabian B. Kugler and Jan von Delft \\ Physics Department, Arnold Sommerfeld Center for Theoretical Physics, and Center for NanoScience, \\ Ludwig-Maximilians-Universität München, Theresienstr. 37, 80333 Munich, Germany
}

(Dated: May 15, 2018)

\begin{abstract}
We study the Fermi-edge singularity, describing the response of a degenerate electron system to optical excitation, in the framework of the functional renormalization group (fRG). Results for the (interband) particle-hole susceptibility from various implementations of fRG (one- and twoparticle-irreducible, multi-channel Hubbard-Stratonovich, flowing susceptibility) are compared to the summation of all leading logarithmic (log) diagrams, achieved by a (first-order) solution of the parquet equations. For the (zero-dimensional) special case of the X-ray-edge singularity, we show that the leading log formula can be analytically reproduced in a consistent way from a truncated, one-loop fRG flow. However, reviewing the underlying diagrammatic structure, we show that this derivation relies on fortuitous partial cancellations special to the form of and accuracy applied to the $\mathrm{X}$-ray-edge singularity and does not generalize.
\end{abstract}

\section{INTRODUCTION}

Fermi-edge singularities describe infrared divergences in optical spectra arising from the discontinuity of the electronic distribution. The advance in the experimental techniques of cavity quantum electrodynamics [1-3] has renewed the need for a precise understanding of such response functions of degenerate Fermi systems to optical excitation. From a theoretical perspective, the study of the X-ray-edge singularity serves as a prototypical fermionic problem which exhibits a logarithmically divergent perturbation theory [4]. Whereas a solution of the (interband) particle-hole susceptibility via parquet equations [5,6] amounts to rather involved computational effort, Lange et al. [7] have recently suggested to perform this resummation via simple approximations in a functional renormalization group (fRG) scheme. Here, we confirm that it is, indeed, possible to reproduce the (firstorder) parquet result from a truncated, one-loop fRG flow without further approximations. However, a detailed analysis of the underlying diagrammatic structure shows that this conclusion relies on fortuitous partial cancellations special to the X-ray-edge singularity.

In more detail, experimentally, X-ray absorption in metals has been a topic of interest for a long time. Similar measurements with infrared light can be performed using heavily doped semiconductors. Whereas photon absorption in metals typically excites a localized deep core electron, effects due to the mobility of valence-band electrons in semiconductors can significantly alter the absorption spectrum [2]. When a quasi-two-dimensional layer of such a semiconducting material is placed inside an optical cavity, the reversible light-matter coupling leads to the formation of half-light, half-matter excitations, attributed to the so-called polariton [8]. Properties of the microcavity system are deduced from the polariton, i.e., from the photon dressed by light-matter interaction, bringing its self-energy into focus [9-11]. To leading order in the coupling, this self-energy is proportional to the particle-hole susceptibility, well-known from the standard literature on the Fermi-edge singularity [5, 6, 12-15].
The effect of light-matter interaction on the photon is thus governed by a correlation function of the fermionic system.

The basic theoretical formulation of the X-ray-edge singularity involves a localized scattering impurity, corresponding to a deep core level of a metal. In this form, the problem is exactly solvable in a one-body approach, as performed by Nozières and De Dominicis [12]. This approach is, however, limited to the special case that the scattering impurity is structureless. If the problem is tackled in a many-body treatment, the solution can be generalized to more complicated situations and has relevance for other problems involving logarithmic divergences. This includes the Kondo problem $[16,17]$ as well as the generalization to scattering processes involving a finite-mass valence-band hole, as necessary for the description of optical absorption in semiconductors $[13,14]$.

In a diagrammatic treatment of the Fermi-edge singularity, logarithmic divergences appear at all orders, demanding resummation procedures. A suitable resummation, containing all leading logarithmic (log) diagrams, can be phrased in terms of parquet equations. These consist of coupled Bethe-Salpeter equations in two-particle channels; here, distinguished by antiparallel or parallel conduction-valence-band lines [5]. Parquet equations can be used in a variety of theoretical applications [18], and it is worthwile to explore whether results comparable or even equivalent to solving those can be obtained by alternative resummation techniques, such as fRG.

The functional renormalization group is a versatile many-body framework, which has proven to give accurate results for low-dimensional fermionic systems [19, 20]. Different realizations and approximations of an exact hierarchy of differential equations for vertex functions allow for rich resummations in the calculation of correlation functions. Inspired by Lange et al. [7], we study the Fermiedge singularity and show that, for the (zero-dimensional) special case of the X-ray-edge singularity, it actually is possible to analytically derive the (first-order) parquet result from a one-loop fRG scheme. However, this derivation relies on fortuitous partial cancellations of diagrams 
and cannot be applied to more general situations. We further show that various truncated fRG flows (see below) do not provide a full summation of parquet diagrams. Though this conclusion may seem disappointing, we believe that the analysis by which it was arrived at is very instructive and motivates the extension of one-loop fRG by multiloop corrections. Indeed, in two follow-up publications [21, 22], we present a multiloop fRG flow that does succeed in summing all parquet diagrams for generic many-body systems.

The paper is organized as follows. In Sec. II, we give the standard formulation of the Fermi-edge and X-ray-edge singularity. The basics of the parquet solution are briefly reviewed in Sec. III, before, in Sec. IV, we introduce the fRG framework in its one-particle- and two-particleirreducible form. In Sec. V, we apply the fRG flow to the fermionic four-point vertex and construct the particle-hole susceptibility at the end of the flow. Furthermore, we briefly consider the potential of computing this susceptibility using a Hubbard-Stratonovich transformation. In Sec. VI, we rephrase the particle-hole susceptibility as a photonic self-energy to obtain a "flowing susceptibility"; we compare results from using a dynamic and static four-point vertex and use the latter approach to analytically reproduce the parquet formula. We also relate our findings to the work by Lange et al. [7] and show how their treatment can be simplified. Finally, we present our conclusions in Sec. VII.

\section{FERMI-EDGE SINGULARITY}

In this section, we review the standard formulation of the Fermi-edge singularity for a two-band electron system. We are interested in the (interband) particle-hole susceptibility, describing the response to optical excitation. A typical absorption process, where a photon lifts an electron from the lower to the upper band, is shown in Fig. 1(a). There, we anticipate the simplification to the X-ray-edge singularity, ignoring kinetic energy in the lower band, thereby considering a static, photo-excited scattering impurity.

Before going into detail, let us state more generally the Hamiltonian of the Fermi-edge singularity,

$$
H^{\prime}=\sum_{k} \epsilon_{k} c_{k}^{\dagger} c_{k}+\sum_{k} E_{k} d_{k}^{\dagger} d_{k}+\frac{U}{V} \sum_{k p q} c_{k+q}^{\dagger} c_{k} d_{p-q}^{\dagger} d_{p}
$$

describing a two-band electron system with interband (screened) Coulomb interaction of the contact type $\left(U_{\boldsymbol{q}}=\right.$ $U>0)$. The operator $c_{\boldsymbol{k}}\left(d_{\boldsymbol{k}}\right)$ annihilates an electron in the conduction (valence) band, $V$ is the volume, and the dispersion relations $\epsilon_{\boldsymbol{k}}, E_{\boldsymbol{k}}$, account for any intraband interaction in a Fermi-liquid picture. This is supposed to work well when electronic energies close to the Fermi level $\mu$, which we take to be on the order of the conductionband width, dominate. Using the effective electron and
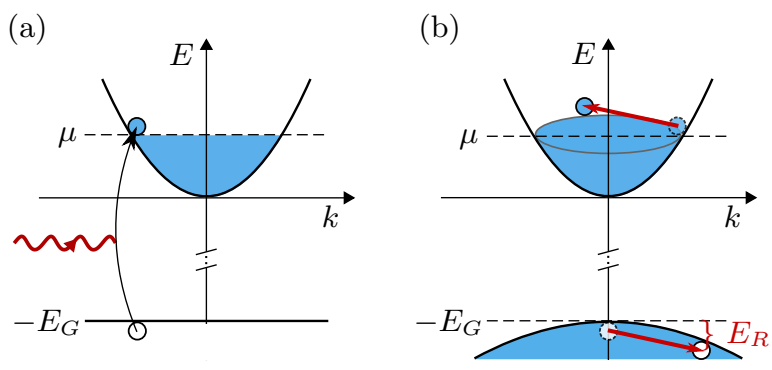

FIG. 1. (Color online) Bandstructure illustrations for twoband electron systems with chemical potential $\mu$ and band gap $E_{G}$. (a) X-ray absorption in metals typically excites a localized, deep core level to the conduction band. The flat band acts as a two-level scattering impurity for conduction electrons. (b) A similar process occurs with infrared light in (direct-gap) heavily doped semiconductors. Only in the limit of infinite valence-band (hole) mass, one reverts to the situation of (a). Accounting for the mobility of the hole, scattering processes of conduction electrons on top of the Fermi surface cost a finite amount of energy, the recoil energy $E_{R}$.

hole masses, $m$ and $m_{h}$, one has $(\hbar=1)$

$$
\epsilon_{\boldsymbol{k}}=\frac{\boldsymbol{k}^{2}}{2 m}, \quad E_{\boldsymbol{k}}=-E_{G}-\frac{\boldsymbol{k}^{2}}{2 m_{h}}, \quad E_{G}>0 .
$$

Note that we further ignore Auger-type interactions containing three $c$ or $d$ operators, since such transitions are suppressed by the size of the band gap $E_{G}$. This allows us to treat electrons from both bands as different fermion species, each with conserved particle number. With the targeted (leading log) accuracy (cf. Sec. III), including spin degeneracy (while keeping the density-density interaction) only results in a doubled density of states $\rho$ [12]. In two space dimensions, the free density of states is $m /(2 \pi)$; in other cases, one approximates $\rho$ by its value at the Fermi level [cf. Eq. (11)].

The particle-hole susceptibility is a two-particle correlation function, given by

$$
i \Pi^{\prime}(\boldsymbol{q}, t)=\frac{1}{V} \sum_{\boldsymbol{k}, \boldsymbol{p}}\left\langle\mathcal{T} d_{\boldsymbol{k}}^{\dagger}(t) c_{\boldsymbol{k}+\boldsymbol{q}}(t) c_{\boldsymbol{p}+\boldsymbol{q}}^{\dagger}(0) d_{\boldsymbol{p}}(0)\right\rangle,
$$

with time-ordering operator $\mathcal{T}$. It exhibits an infrared divergence - the Fermi-edge singularity - which is cut by the (valence-band) recoil energy $[11,13]$ at Fermi momentum, equal to $\mu \cdot m / m_{h}$ [cf. Fig. 1(b)].

For the case of a polariton experiment using, e.g., a GaAs semiconductor [2], one has a ratio of effective masses between the conduction and heavy-hole-valence band [11] of $m / m_{h} \sim 0.14$. Considering X-ray absorption in metals, one usually encounters the excitation of a localized, deep core level to the conduction band [cf. Fig. 1(a)]. This motivates the severe simplification of an infinite valenceband (hole) mass, corresponding to a two-level scattering impurity, resulting in the Hamiltonian known from the X-ray-edge singularity, $\left(\epsilon_{d}=-E_{G}<0\right)$

$$
H=\sum_{k} \epsilon_{\boldsymbol{k}} c_{\boldsymbol{k}}^{\dagger} c_{\boldsymbol{k}}+\epsilon_{d} d^{\dagger} d+\frac{U}{V} \sum_{\boldsymbol{k} \boldsymbol{p}} c_{\boldsymbol{k}}^{\dagger} c_{\boldsymbol{p}} d^{\dagger} d
$$


Momentum dependencies in interband quantities are completely absorbed by the infinitely heavy hole, and only the local conduction-band operators play a role:

$$
i \Pi(t)=\left\langle\mathcal{T} d^{\dagger}(t) c(t) c^{\dagger}(0) d(0)\right\rangle, \quad c=\frac{1}{\sqrt{V}} \sum_{\boldsymbol{k}} c_{\boldsymbol{k}} .
$$

Without the intrinsic infrared cutoff of the recoil energy, the (infinite-mass) particle-hole susceptibility shows a true divergence. In a zero-temperature calculation and for small interaction, this takes the form $[5,6,12]$

$$
\Pi(\omega)=\frac{\rho}{2 u}\left[1-\left(\frac{\omega+\xi_{d}+i 0^{+}}{-\xi_{0}}\right)^{-2 u}\right], \quad u=\rho U .
$$

Here, $-\xi_{d}=\mu-\epsilon_{d}=\mu+E_{G}$ is the threshold frequency and $\xi_{0} \sim \mu$ an intrinsic ultraviolet cutoff of the order of the conduction-band width [cf. Eq. (11)]. Note that, for absorption processes, one has an initially fully occupied valence band $\left(E_{G} \gg k_{B} T\right)$, such that $\Pi(t)$ is automatically retarded. Analogously, the valence-band propagator $i G^{d}(t)=\left\langle\mathcal{T} d(t) d^{\dagger}\right\rangle$ is purely advanced. Although our calculations will proceed in a finite-temperature formalism, we aim to reproduce the result (6). Hence, we numerically consider very low temperatures and perform the zero-temperature limit in analytic calculations. As we attribute the constant Hartree part of a fermionic selfenergy to the renormalized band gap $E_{G}$, a diagrammatic expansion using $G^{d}(t) \propto \Theta(-t)$ (with the Heaviside step function) directly shows that conduction-band propagators are not further renormalized by interband interaction.

As already mentioned, the particle-hole susceptibility can also be viewed as the leading contribution (in the light-matter coupling $\rho|M|^{2}, M$ being the dipole matrix element) to a photon self-energy. In the regime under consideration, electronic processes happen on a timescale $1 / \mu$ much shorter than typical times of absorption and emission of a photon $1 /\left(\rho|M|^{2}\right)$ [11]. For $\mu \gg \rho|M|^{2}$, one can thus approximate the photon self-energy by an interacting particle-hole bubble, given the standard coupling

$$
H_{\mathrm{cpl}}^{\prime}=\frac{1}{\sqrt{V}} \sum_{\boldsymbol{p} \boldsymbol{q}}\left(M c_{\boldsymbol{p}+\boldsymbol{q}}^{\dagger} d_{\boldsymbol{p}} a_{\boldsymbol{q}}+M^{*} d_{\boldsymbol{p}}^{\dagger} c_{\boldsymbol{p}+\boldsymbol{q}} a_{\boldsymbol{q}}^{\dagger}\right),
$$

where $a_{\boldsymbol{q}}$ annihilates a photon. For infinite hole mass, the momentum dependence of the photon absorption can no longer be resolved, and we use the simplified coupling

$$
H_{\mathrm{cpl}}=M c^{\dagger} d a+M^{*} d^{\dagger} c a^{\dagger}, \quad \sum_{\boldsymbol{q}} a_{\boldsymbol{q}}=a .
$$

Having defined the system under consideration [Eq. (4)] and the quantity of interest [Eq. (5)], our analysis will proceed in an imaginary-time action formalism. We transform the Grassmann fields for both bands $(c, \bar{c}, d, \bar{d})$ to Matsubara frequencies according to $c_{\omega}=$ $\int_{0}^{\beta} \mathrm{d} \tau c(\tau) e^{i \omega \tau} / \sqrt{\beta}$, etc., where $\beta=1 /\left(k_{B} T\right)$. For the X-ray-edge singularity, a change to the position basis immediately shows that conduction-band fields other than the local ones [cf. Eq. (5)] can be integrated out, leading to the action

$$
\begin{aligned}
S & =-\int_{\omega} G_{0, \omega}^{c,-1} \bar{c}_{\omega} c_{\omega}-\int_{\omega} G_{0, \omega}^{d,-1} \bar{d}_{\omega} d_{\omega} \\
& +U \int_{\omega \nu \bar{\omega}}^{\prime} \bar{d}_{\omega} d_{\nu} \bar{c}_{\bar{\omega}+\nu} c_{\bar{\omega}+\omega} .
\end{aligned}
$$

Here, we have introduced a notation where $\int_{\omega}$ is a sum over Matsubara frequencies, bosonic Matsubara frequencies are denoted by a bar, and each prime on an integral sign represents a prefactor of $1 / \beta$. The zero-temperature limit is then conveniently obtained as

$$
\lim _{\beta \rightarrow \infty} \int_{\omega}^{\prime} f_{\omega}=\int \frac{\mathrm{d} \omega}{2 \pi} f(\omega) .
$$

It is worth noting that the action of the more general Fermi-edge singularity, defined by the Hamiltonian (1), is perfectly analogous to the one of the X-ray-edge singularity [Eq. (9)]. One merely has to identify each Matsubara frequency with a double index for frequency and momentum $(\omega, \boldsymbol{k})$ and Matsubara summations with a double sum over frequencies and momenta, the prefactor being $1 /(\beta V)$ instead of $1 / \beta$. Hence, all diagrammatic and fRG arguments apply simultaneously to the case of finite and infinite hole mass. Only for numerical as well as analytic computations, we restrict ourselves to the (zero-dimensional) special case of the X-ray-edge singularity, such that we can readily ignore any momentum dependence.

Whereas for finite hole mass, the propagator of valence (conduction) electrons is given by $1 /\left(i \omega+\mu-E_{\boldsymbol{k}}\right)$ $\left[1 /\left(i \omega+\mu-\epsilon_{\boldsymbol{k}}\right)\right]$, for infinite mass, the valence-band propagator simply reads $G_{0, \omega}^{d}=1 /\left(i \omega-\xi_{d}\right)$. As we use a parabolic dispersion in the conduction band, we introduce an ultraviolet cutoff $\epsilon_{\boldsymbol{k}} \leq \mu+\xi_{0}$ in momentum space. The choice of a half-filled conduction band, i.e., $\xi_{0}=\mu$, yields the particularly simple local propagator

$$
\begin{gathered}
G_{0, \omega}^{c}=\frac{1}{V} \sum_{\boldsymbol{k}} \frac{1}{i \omega-\epsilon_{\boldsymbol{k}}+\mu}=\rho \int_{-\xi_{0}}^{\xi_{0}} \mathrm{~d} \xi \frac{1}{i \omega-\xi} \\
=-2 i \rho \arctan \left(\xi_{0} / \omega\right) \approx-i \pi \rho \operatorname{sgn}(\omega) \Theta\left(\xi_{0}-|\omega|\right) .
\end{gathered}
$$

In the last step, we have ignored any details of the ultraviolet cutoff, which are of no physical relevance. Note that different leading log diagrams typically contain the energy range of occupied $(\mu)$ or unoccupied conduction band states $\left(\xi_{0}\right)$ in the argument of the logarithm. Minor deviations from half-filling, still in the regime of $\left|\mu-\xi_{0}\right| \ll \xi_{0}$, have only subleading effects.

Including photon fields $(a, \bar{a})$ into the theory, one might perform a simple transformation for dimensional reasons of the type $\gamma=M a, \bar{\gamma}=M^{*} \bar{a}$, resulting in a rescaled coupling term

$$
S_{\mathrm{cpl}}=\frac{1}{\sqrt{\beta}} \int_{\bar{\omega} \omega}\left(\bar{c}_{\bar{\omega}+\omega} d_{\omega} \gamma_{\bar{\omega}}+\bar{d}_{\omega} c_{\bar{\omega}+\omega} \bar{\gamma}_{\bar{\omega}}\right) .
$$




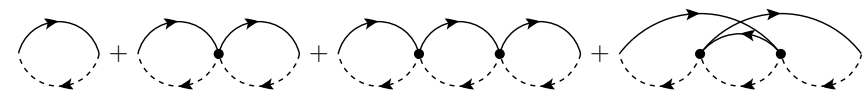

FIG. 2. Particle-hole susceptibility П [Eq. (13)] up to second order in the interaction, consisting of the first three ladder diagrams $[\mathrm{L}(0), \mathrm{L}(1), \mathrm{L}(2)]$ and the crossed diagram $[\mathrm{C}(2)]$. Full (dashed) lines denote propagators of conduction (valence) electrons. Dots represent bare vertices with a factor $-U$.

Then, in the limit of $M \rightarrow 0$, i.e., $G_{0}^{\gamma} \propto|M|^{2} \rightarrow 0$, one obtains the leading contribution to the photon self-energy $\Pi^{\gamma}$ as precisely the particle-hole susceptibility

$$
\lim _{M \rightarrow 0} \Pi_{\bar{\omega}}^{\gamma}=\Pi_{\bar{\omega}}=\int_{\omega \nu}^{\prime}\left\langle\bar{d}_{\omega} d_{\nu} \bar{c}_{\bar{\omega}+\nu} c_{\bar{\omega}+\omega}\right\rangle
$$

Again, the formula is similarly applicable for the more general Fermi-edge singularity, where $\bar{\omega}$ denotes frequency and momentum $(\bar{\omega}, \boldsymbol{q})$. According to the rules of analytic continuation, $i \bar{\omega} \rightarrow \omega+i 0^{+}$, the X-ray-edge singularity written in terms of Matsubara frequencies can directly be inferred from Eq. (6):

$$
\Pi_{\bar{\omega}}=\frac{\rho}{2 u}\left[1-\left(\frac{i \bar{\omega}+\xi_{d}}{-\xi_{0}}\right)^{-2 u}\right] .
$$

It is our goal to reproduce this result, originating from a (first-order) solution of the parquet equations, using an fRG scheme. Before getting into the details of fRG, let us briefly review the basics of the parquet solution leading to Eq. (14).

\section{FIRST-ORDER PARQUET SOLUTION}

We already mentioned that the X-ray-edge singularity has been exactly solved in a one-body approach [12] containing the parquet result (6) in the weak-coupling limit. For the sake of generalizability to actual fermionic manybody problems, one is interested in other (approximate) solutions obtained from a many-body treatment. Roulet et al. [5] have achieved such a solution of the X-ray-edge singularity in leading order of the logarithmic singularity. This first-order parquet solution sums up all perturbative terms of the type $u^{n+p} \ln ^{n+1}\left|\xi_{0} /\left(\omega+\xi_{d}\right)\right|$, where $p=0$. These correspond to the leading log (or parquet) diagrams; subleading terms with $p>0$ are neglected. Such an approximation is applicable for small interaction, $u \ll 1$, and frequencies not too close to the threshold $-\xi_{d}$. Yet, a subsequent work [6] as well as the exact solution [12] show that, for small coupling, the result actually holds for frequencies arbitrarily close to the threshold.

The lowest-order diagrams for the particle-hole susceptibility, corresponding to the first terms of an expansion of Eq. (14) in $u$, are shown in Fig. 2. Full lines denote conduction-band $(c)$ and dashed lines valence-band $(d)$ propagators. Self-energy corrections, affecting the $d$ propagator, can be ignored, as discussed later. A bare vertex, symbolized by a solid circle, demands energy(-momentum)

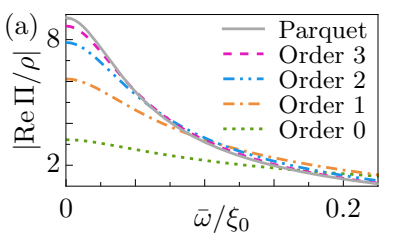

(b)

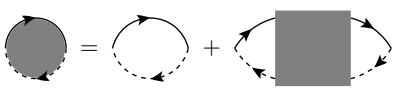

FIG. 3. (a) (Color online) Leading log formula in terms of Matsubara frequencies [Eq. (14)] at increasing orders in the coupling $u$. Numerical parameters are $u=0.28,\left|\xi_{d} / \xi_{0}\right|=$ $1 / 25$, and the grid for Matsubara frequencies is set by $\beta \xi_{0}=$ 500. (The same parameters are used throughout this work.) Here, we show (connected) lines for clarity. (b) The particlehole susceptibility $\Pi$ (full circle) can be expressed via the bare bubble and the 1PI four-point vertex $\Gamma^{(4)}$, denoted by a full square, according to Eq. (16).

conservation and multiplication by $-U$. Apart from that, there are no combinatorial or sign factors attached to diagrams. Free variables are to be integrated over with dimension-full integrals [cf. Eq. (10)].

The first three diagrams in Fig. 2 are called ladder diagrams. It is easy to see that taking into account only ladder diagrams leads to the false prediction of a bound state [14]. Crossed diagrams, such as the last diagram in Fig. 2, are crucial for an accurate description and encode screening effects (conduction-band holes) of the Fermi sea. Figure 3(a) shows how the leading log result is built up in an expansion of Eq. (14), exemplified by the real part. Numerical results in Sec. V and Sec. VI aim to reproduce this form. Note that, written in terms of Matsubara frequencies, the particle-hole susceptibility (14) is no longer singular. The seemingly quick convergence of the perturbative curves to the full solution at an interaction parameter $u=0.28$ in Fig. 3(a) is also due to a rapid decay of the expansion coefficients.

Though, for real frequencies, $\xi_{d}$ acts as a frequency shift, it is a property of the analytic continuation that, in imaginary-frequency space, different values for $\xi_{d}$ stretch/flatten the curve. Since we have incorporated the physical effect of the size of the band gap already in the choice of the interaction in the Hamiltonian (1), we can choose any value for $\xi_{d}$ in our calculations. In order to have a pronounced peak in the Matsubara curve, we take $\left|\xi_{d} / \xi_{0}\right|=1 / 25$, implying $u \ln \left|\xi_{0} / \xi_{d}\right| \approx 0.9$. Note that, as can be seen from the simple computation of the particle-hole bubble, zero-temperature calculations are discontinuous w.r.t. to $\xi_{d}$ at $\xi_{d}=0$. Choosing $\xi_{d}=0$, one loses analytic properties and only obtains the real part of the logarithmic factors depending on $|\bar{\omega}|$ (cf. App.).

The four-point correlation function in the particle-hole susceptibility can be rephrased by cutting external legs (in general, as dressed propagators $G^{d}, G^{c}$ ) in the connected part according to [cf., e.g., Eq. (6.92) of Ref. 20]

$$
\begin{aligned}
\left\langle\bar{d}_{\omega} d_{\nu} \bar{c}_{\bar{\omega}+\nu} c_{\bar{\omega}+\omega}\right\rangle= & G_{\omega}^{d} G_{\bar{\omega}+\omega}^{c} \delta_{\omega, \nu}+G_{\omega}^{d} G_{\nu}^{d} \\
& \times G_{\bar{\omega}+\omega}^{c} G_{\bar{\omega}+\nu}^{c} \Gamma_{\omega, \bar{\omega}+\omega, \bar{\omega}+\nu, \nu}^{\bar{d} c \bar{c} d} / \beta .
\end{aligned}
$$




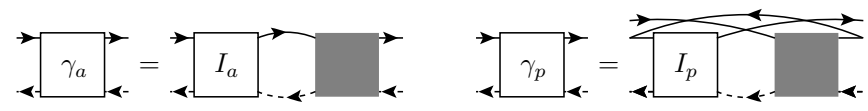

FIG. 4. Bethe-Salpeter equations for both two-particle channels, where $\gamma_{a}$ and $\gamma_{p}$ are reducible while $I_{a}$ and $I_{p}$ are irreducible vertices in antiparallel and parallel conduction-valenceband lines, respectively. The vertices are further related via Eq. (17a).

This introduces the one-particle-irreducible (1PI) fourpoint vertex $\Gamma^{\bar{d} c \bar{c} d}$. Consequently, the particle-hole susceptibility is fully determined by $\Gamma^{(4)}=\Gamma^{\bar{d} c \bar{c} d}$ via

$$
\Pi_{\bar{\omega}}=\int_{\omega}^{\prime} G_{\omega}^{d} G_{\bar{\omega}+\omega}^{c}+\int_{\omega \nu}^{\prime \prime} G_{\omega}^{d} G_{\nu}^{d} G_{\bar{\omega}+\omega}^{c} G_{\bar{\omega}+\nu}^{c} \Gamma_{\omega, \bar{\omega}+\omega, \bar{\omega}+\nu, \nu}^{(4)}
$$

the graphical representation of which is shown in Fig. 3(b).

The parquet equations are then focused on the fourpoint vertex and use a diagrammatic decomposition in two-particle channels. For the Fermi-edge singularity, the leading log divergence is determined by the two channels characterized by parallel and antiparallel conductionvalence-band lines:

$$
\begin{gathered}
\Gamma^{(4)}=R+\gamma_{p}+\gamma_{a}, \quad I_{p}=R+\gamma_{a}, \quad I_{a}=R+\gamma_{p}, \\
\gamma_{a ; \omega, \bar{\omega}+\omega, \bar{\omega}+\nu, \nu}=\int_{\omega^{\prime}}^{\prime} I_{a ; \omega, \bar{\omega}+\omega, \bar{\omega}+\omega^{\prime}, \omega^{\prime}} G_{\omega^{\prime}}^{d} G_{\bar{\omega}+\omega^{\prime}}^{c} \\
\quad \times \Gamma_{\omega^{\prime}, \bar{\omega}+\omega^{\prime}, \bar{\omega}+\nu, \nu}^{(4)}, \\
\gamma_{p ; \omega, \bar{\nu}-\nu, \bar{\nu}-\omega, \nu}=\int_{\omega^{\prime}}^{\prime} I_{p ; \omega, \bar{\nu}-\omega^{\prime}, \bar{\nu}-\omega, \omega^{\prime}} G_{\omega^{\prime}}^{d} G_{\bar{\nu}-\omega^{\prime}}^{c} \\
\quad \times \Gamma_{\omega^{\prime}, \bar{\nu}-\nu, \bar{\nu}-\omega^{\prime}, \nu}^{(4)} .
\end{gathered}
$$

Here, $R$ is the totally (two-particle-) irreducible vertex; $\gamma_{a}$ and $\gamma_{p}$ are reducible while $I_{a}$ and $I_{p}$ are irreducible vertices in the antiparallel and parallel channel, respectively. Note that a $\Gamma^{(4)}$ diagram can be reducible in exclusively one of the two channels [5]; diagrams irreducible in both channels belong to $R$. The Bethe-Salpeter equations for $\gamma_{a}(17 \mathrm{~b})$ and $\gamma_{p}(17 \mathrm{c})$, which are the crucial components of the parquet equations, are illustrated in Fig. 4.

The parquet equations (17) as such are exact and merely represent a classification of diagrams. In the first-order solution [5] (also referred to as parquet approximation [18]), one approximates the totally irreducible vertex by its bare part, i.e., $R=-U$. To be consistent with the leading $\log$ summation (of the X-ray-edge singularity), one further neglects any fermionic self-energies $[5,6]$. In fact, it is easily shown that the lowest (non-constant) contribution to $\Sigma^{d}$ involves the subleading term $u^{2} \ln \left|\xi_{0} /\left(\omega+\xi_{d}\right)\right|$. Similarly, higher-order corrections to $R$ are subleadingly divergent. From the exact solution [12], it is known that extensions of the first-order parquet scheme just lead to the replacement of $u$ by more complicated functions of $u$ in the characteristic form of the particle-hole susceptibility [Eq. (6)]. For weak coupling, it is thus justified

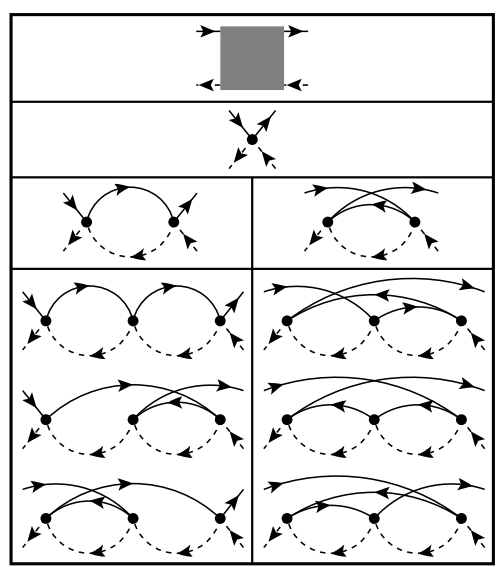

FIG. 5. Parquet graphs for the four-point vertex $\Gamma^{(4)}$, consisting of diagrams reducible in (left) antiparallel lines and (right) parallel lines, up to third order in the interaction. Note that all diagrams are obtained by successively replacing bare vertices by antiparallel and parallel bubbles.

to focus on the leading-order result. We will henceforth ignore all fermionic self-energies and omit the index 0 on fermionic propagators when referring to the X-ray-edge singularity. (It should be noted that these arguments do not directly apply to any Fermi-edge singularity. In particular, considering a finite-mass valence-band hole, it was shown that $\Sigma^{d}$ has a crucial effect on the particlehole susceptibility and encodes the influence of indirect transitions $[11,13]$.)

From the parquet equations (17), one can also extract the diagrammatic content of the emergent four-point vertex $\Gamma^{(4)}$. All leading log diagrams (parquet graphs) are obtained by successively replacing bare vertices (starting from the first-order, bare vertex) by parallel and antiparallel bubbles (cf. Fig. 5). Note that such a parquet resummation is the natural extension to two channels of what the ladder summation is to one channel. Having gained insight into the structure of the parquet equations and the leading log diagrams, let us move on to the formalism used in the remainder of this paper.

\section{FUNCTIONAL RENORMALIZATION GROUP}

The functional renormalization group (fRG) is a manybody framework, which in principle allows one to examine the renormalization group flow of all coupling constants in their full functional dependence and to obtain diagrammatic resummations of vertex and correlation functions. Its basic idea is to consider the change of a many-body generating functional upon the variation of an artificially introduced scale parameter, which can act as an effective infrared cutoff and allows to successively integrate out high-energy degrees of freedom. This procedure of "zooming out" from microscopic to many-body physics, i.e., the evolution of physical quantities upon lowering the scale 


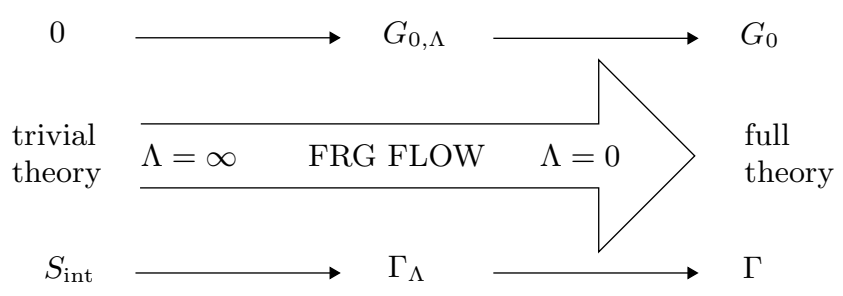

FIG. 6. 1PI fRG flow: The flow parameter $\Lambda$, introduced in the quadratic part of the action, makes the theory evolve from a trivial to the original, full one. At the initial scale, the (quantum) effective action $\Gamma$ can directly be read off from the interacting part of the action $S_{\text {int }}$. Finally, the desired generating functional for $1 \mathrm{PI}$ vertices $\Gamma$ is obtained.

parameter $\Lambda$, modulating from a trivial to the full theory (cf. Fig. 6), is described by an exact functional differential equation.

Most commonly, one incorporates the scale parameter in the bare propagator of the theory. Since we are interested in interband quantities such as the particle-hole susceptibility, it is sufficient to modify the propagator of one band alone. As $G_{0, \omega}^{d}$ follows the typical $1 /\left(i \omega-\xi_{d}\right)$ behavior (cf. Sec. II), it is convenient to choose the lower band. The appropriate boundary conditions, to initially $\left(\Lambda_{i}=\infty\right)$ extinguish all interband diagrams and finally $\left(\Lambda_{f}=0\right)$ revert to the original theory, are $G_{0, \Lambda_{i}}^{d}=0$, $G_{0, \Lambda_{f}}^{d}=G_{0}^{d}$.

We will use two alternative realizations with particularly useful computational properties, namely the $\delta$ regulator,

$$
\begin{gathered}
G_{0, \Lambda, \omega}^{d}=\Theta(|\omega|-\Lambda) G_{0, \omega}^{d}=\frac{\Theta(|\omega|-\Lambda)}{i \omega-\xi_{d}}, \\
\partial_{\Lambda} G_{0, \Lambda, \omega}^{d}=-\delta(|\omega|-\Lambda) G_{0, \omega}^{d}=\frac{-\delta(|\omega|-\Lambda)}{i \omega-\xi_{d}},
\end{gathered}
$$

and the Litim [23] regulator,

$$
\begin{aligned}
G_{0, \Lambda, \omega}^{d} & =\frac{1}{i \operatorname{sgn}(\omega) \max (|\omega|, \Lambda)-\xi_{d}}, \\
\partial_{\Lambda} G_{0, \Lambda, \omega}^{d} & =\frac{-i \operatorname{sgn}(\omega) \Theta(\Lambda-|\omega|)}{\left[i \operatorname{sgn}(\omega) \Lambda-\xi_{d}\right]^{2}} .
\end{aligned}
$$

In an exact solution of the flow, all regulators give identical results since, at the end of the flow $\left(\Lambda_{f}=0\right)$, the original theory is restored. However, once approximations are made, the outcomes might differ significantly. In particular, this will happen once the flow of certain quantities does not form a total derivative of diagrams, e.g., due to truncation.

One can consider different functionals paraphrasing the many-body problem under the fRG flow. Two common choices are the (quantum) effective action and the Luttinger-Ward functional serving as generating functionals for one-particle-irreducible (1PI) and two-particleirreducible (2PI) vertices, respectively. Our study is focused on 1PI fRG flows. We will only briefly mention the
2PI formulation to show that this provides no benefit for our treatment.

\section{A. One-particle-irreducible formulation}

The (quantum) effective action $\Gamma$ is obtained from the (log of the) partition function - in the presence of sources coupled directly to the fields $\left(S_{\mathrm{src}}=\int_{\alpha} j_{\alpha} \varphi_{\alpha}\right)$-by a Legendre transformation. Its behavior under the flow is given by the (so-called) Wetterich equation [24]. In the notation of Ref. 20, particularly useful for mixed (fermionic and bosonic) theories, it is stated as

$$
\begin{aligned}
\partial_{\Lambda} \Gamma_{\Lambda}[\bar{\varphi}] & =-\frac{1}{2} \operatorname{STr}\left\{\left(\partial_{\Lambda} G_{0, \Lambda}^{-1}\right)\right. \\
& \left.\times\left(\left[\left(\frac{\delta^{2} \Gamma_{\Lambda}[\bar{\varphi}]}{\delta \bar{\varphi} \delta \bar{\varphi}}\right)^{\mathrm{T}}-G_{0, \Lambda}^{-1}\right]^{-1}+G_{0, \Lambda}\right)\right\} .
\end{aligned}
$$

Here, the super trace runs over multi-indices $\alpha$, which specify field as well as conjugation indices and all further quantum numbers, and contains a minus sign when summing over fermionic degrees of freedom. If the propagator of all fields is set to zero at the beginning of the flow, the initial condition for $\Gamma$ is given by the interacting part of the action [20], $\Gamma_{\Lambda_{i}}=S_{\mathrm{int}}$ (no renormalization of vertices by propagating degrees of freedom is possible). Although we choose only the bare valence-band propagator to be $\Lambda$-dependent, all interband quantities are still given by the bare interactions of $S_{\text {int }}$.

In order to tackle the fundamental and in general unsolvable flow equation (20), $\Gamma$ can be expanded in terms of $1 \mathrm{PI} n$-point vertices $\Gamma^{(n)}$, where we set

$$
\Gamma_{\alpha_{1} \ldots \alpha_{n}}^{(n)}=\left.\beta^{\frac{n}{2}-1} \frac{\delta^{n} \Gamma[\bar{\varphi}]}{\delta \bar{\varphi}_{\alpha_{1}} \ldots \delta \bar{\varphi}_{\alpha_{n}}}\right|_{\bar{\varphi}=0} .
$$

The functional differential equation (20) is transformed into a hierarchy of infinitely many coupled ordinary differential equations with an interesting structure [20]: $\partial_{\Lambda} \Gamma^{(n)}$ depends on other vertices only up to $\Gamma^{(n+2)}$ and, then, always via $\mathrm{S} \operatorname{Tr}\left\{\Gamma^{(n+2)} S\right\}$. Here, $S$ is the (so-called) single-scale propagator $S=-G\left(\partial_{\Lambda} G_{0}^{-1}\right) G$, adding selfenergy corrections to a differentiated bare line. Since, with logarithmic accuracy (cf. Sec. III), we can neglect fermionic self-energies, we have the notable simplification $S=\partial_{\Lambda} G_{0}$.

The most common truncation of the still unsolvable hierarchy of flow equations is to leave higher-order vertices constant $\left(\Gamma_{\Lambda}^{n>n_{0}}=\Gamma_{\Lambda_{i}}^{n>n_{0}}\right)$ yielding a finite set of differential equations. This has a weak coupling motivation, as higher-order vertices typically are of increasing order in the interaction. Furthermore, for a four-point interaction as in our fermionic theory, the only non-zero initial condition of a 1PI interband vertex is $\Gamma^{\bar{d} c \bar{c} d}=-U$. Note that, when specifying a vertex, we usually omit the superscript 
(a)

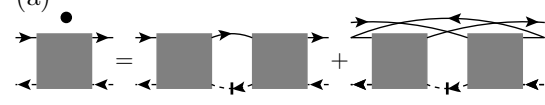

(b)

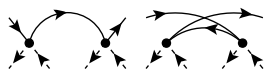

FIG. 7. (a) Diagrammatic representation of the flow equation (22) for $\Gamma^{\bar{d} c \bar{c} d}$ upon neglecting the six-point vertex. The dot denotes the differentiated vertex; lines with a vertical dash symbolize the single-scale propagator. (b) Three-particle vertices $\Phi^{\bar{d} \bar{c} d c d \bar{d}}$ and $\Phi^{\bar{d} c \bar{c} d d \bar{d}}$, responsible for the $2 \mathrm{PI}$ fRG flow of $I_{p}$ and $I_{a}$, respectively, at second order in $U$.

$(n)$ and, instead, write field indices as superscripts and quantum numbers as indices. With the photon included in the theory, we have the additional non-trivial initial condition $\Gamma_{\Lambda_{i}, \omega, \omega-\bar{\omega}, \bar{\omega}}^{\bar{c} d \gamma}=1=\Gamma_{\Lambda_{i}, \omega-\bar{\omega}, \omega, \bar{\omega}}^{\bar{d} c \bar{\gamma}}$ for the mixed three-point vertex.

The flow equations of the individual vertices are obtained by performing the vertex expansion (21) on both sides of the Wetterich equation (20). Given a certain truncation and the above mentioned initial conditions, the set of differential equations can be solved by standard methods, possibly requiring further approximations. Solutions for the self-energy $\left[\Gamma^{(2)}\right]$ or higher-order vertex functions $\left[\Gamma^{(n>2)}\right]$ can be used to compute correlation functions, such as the particle-hole susceptibility [cf. Eq. (16)].

For future reference, let us already state the 1PI fRG flow equation for the four-point vertex in the purely fermionic theory [in the matrix notation of Eq. (20), we omit the second index for one-particle quantities: $G_{\omega, \omega}^{c \bar{c}}=G_{\omega}^{c}$, etc.]. To describe the leading logarithmic divergence of the Fermi-edge singularity, we only consider interband combinations of four-point vertices and obtain

$$
\begin{aligned}
\partial_{\Lambda} & \Gamma_{\Lambda, \omega, \bar{\omega}+\omega, \bar{\omega}+\nu, \nu}^{\bar{d} c} \bar{c} d \\
\times & \int_{\omega^{\prime}}^{\prime} S_{\Lambda, \omega^{\prime}}^{d} \\
\times & \left(\Gamma_{\Lambda, \omega, \bar{\omega}+\omega, \bar{\omega}+\omega^{\prime}, \omega^{\prime}}^{\bar{d} c \bar{\omega} d} G_{\bar{\omega}+\omega^{\prime}}^{c} \Gamma_{\Lambda, \omega^{\prime}, \bar{\omega}+\omega^{\prime}, \bar{\omega}+\nu, \nu}^{\bar{d} c \bar{c} d}\right. \\
& +\Gamma_{\Lambda, \omega, \bar{\nu}-\omega^{\prime}, \bar{\nu}-\omega, \omega^{\prime}}^{\bar{d} c \bar{\nu}} G_{\bar{\nu}-\omega^{\prime}}^{c} \Gamma_{\Lambda, \omega^{\prime}, \bar{\nu}-\nu, \bar{\nu}-\omega^{\prime}, \nu}^{\bar{d} c \bar{c} d} \\
& \left.+\Gamma_{\Lambda, \omega, \bar{\omega}+\omega, \bar{\omega}+\nu, \nu, \omega^{\prime}, \omega^{\prime}}^{\bar{d} c \bar{c} d}\right), \quad \bar{\nu}=\bar{\omega}+\omega+\nu .
\end{aligned}
$$

Without fermionic self-energies, the propagators $G^{c}$, $G^{d}$, and $S^{d}$ are known functions. If the fRG hierarchy is further truncated by discarding the six-point vertex, $\Gamma_{\Lambda}^{(6)}=\Gamma_{\Lambda_{i}}^{(6)}=0$, the resulting flow equation is closed in itself and can be solved as such. Figure 7(a) illustrates this flow equation, where we denote a single-scale propagator, i.e., a differentiated $d$ line, by a vertical dash next to the arrow. Evidently, the 1PI fRG scheme does not yield separate flow equations for four-point vertices distinguished in two-particle channels, in contrast to the parquet equations (17). However, one immediately sees in Fig. 7(a) that contributions from the first summand are reducible in antiparallel lines, whereas contributions from the second one are reducible in parallel lines. Totally irreducible diagrams are still present in Eq. (22) as initial condition (the bare vertex) and encoded in $\Gamma^{(6)}$, but, importantly, contributions from $\operatorname{STr}\left\{\Gamma^{(6)} S\right\}$ are also relevant for higher-order parquet diagrams in both channels (cf. Sec. V). To explore the possibility of treating the two-particle channels separately from the outset, let us sketch the applicability of $2 \mathrm{PI}$ fRG to the Fermi-edge singularity.

\section{B. Two-particle-irreducible formulation}

The $2 \mathrm{PI}$ formulation of fRG is based on the LuttingerWard functional $\Phi$, obtained by a Legendre transformation from the (log of the) partition function with sources coupled to two fields $\left(S_{\mathrm{src}}=\int_{\alpha \alpha^{\prime}} \varphi_{\alpha} J_{\alpha \alpha^{\prime}} \varphi_{\alpha^{\prime}}\right)$. It can be shown [25] and is intuitive from its diagrammatic expansion that, contrary to $\Gamma, \Phi$ does not explicitly depend on the bare propagator of the theory. The scale dependence is only given by its argument $\mathcal{G}$, representing the full propagator. Therefore, one immediately derives the flow equations

$$
\begin{aligned}
\partial_{\Lambda} \Phi[\mathcal{G}] & =\frac{1}{2} \operatorname{STr}\left\{\frac{\delta \Phi}{\delta \mathcal{G}} \partial_{\Lambda} \mathcal{G}\right\}, \\
\partial_{\Lambda} \Phi_{\Lambda, \alpha_{1} \alpha_{1}^{\prime} \ldots \alpha_{n} \alpha_{n}^{\prime}}^{(2 n)} & =\frac{1}{2 \beta} \sum_{\tilde{\alpha}, \tilde{\alpha}^{\prime}} \Phi_{\Lambda, \alpha_{1} \alpha_{1}^{\prime} \ldots \alpha_{n} \alpha_{n}^{\prime} \tilde{\alpha} \tilde{\alpha}^{\prime}}^{(2 n+2)} \partial_{\Lambda} G_{\tilde{\alpha} \tilde{\alpha}^{\prime}}
\end{aligned}
$$

where $G$ is the physical propagator $\left.\mathcal{G}\right|_{J=0}$. Equation (23a) has a much simpler structure compared to the Wetterich equation (20). The 2PI $n$-particle vertices, as coefficients of $\Phi$ when expanded around the physical propagator,

$$
\Phi_{\alpha_{1} \alpha_{1}^{\prime} \ldots \alpha_{n} \alpha_{n}^{\prime}}^{(2 n)}=\left.\beta^{n-1} \frac{\delta^{n} \Phi}{\delta \mathcal{G}_{\alpha_{1} \alpha_{1}^{\prime}} \ldots \delta \mathcal{G}_{\alpha_{n} \alpha_{n}^{\prime}}}\right|_{\mathcal{G}=G}
$$

are primarily suited (to compute correlation functions) for a purely fermionic theory, where vertices only connect an even number of fields.

Unlike the totally antisymmetric 1PI four-point vertex (where particularly $\Gamma^{\bar{d} c \bar{c} d}=\Gamma^{\bar{d} \bar{c} d c}$ ), we have $\Phi^{\bar{d} \bar{c} d c}=I_{p}$ and $\Phi^{\bar{d} c \bar{c} d}=I_{a}$, implying the desired distinction between the two-particle channels. (Note that the parquet approximation, which considers only the bare vertex as the totally irreducible contribution in $I_{p}$ and $I_{a}$ has not yet been made.) In contrast to the parquet equations, the $2 \mathrm{PI}$ flow, however, does not interrelate these two-particle vertices; instead, it demands the computation of corresponding three-particle vertices. Moreover, since the 2PI vertices $\Phi^{(2 n)}$ are not necessarily 1PI, their initial conditions are more complex than those of $\Gamma^{(n)}$ : We have $\Phi_{\Lambda_{i}}^{(2 n)} \neq 0$ for infinitely many $n$, namely for all $\Phi^{(2 n)}$ which contain diagrams without internal valence-band lines [cf. Fig. 7(b)]. Therefore, truncation schemes need to be devised more carefully in the 2PI formulation.

The flow equations for $I_{p}$ and $I_{a}$, deduced from 
Eq. (23b),

$$
\begin{aligned}
& \partial_{\Lambda} I_{p ; \omega_{1}, \omega_{2}, \omega_{3}, \omega_{4}}=\int_{\omega}^{\prime} \Phi_{\omega_{1}, \omega_{2}, \omega_{3}, \omega_{4}, \omega, \omega}^{\bar{d} \bar{c} d c d \bar{\Lambda}} G_{\omega}^{d}, \\
& \partial_{\Lambda} I_{a ; \omega_{1}, \omega_{2}, \omega_{3}, \omega_{4}}=\int_{\omega}^{\prime} \Phi_{\omega_{1}, \omega_{2}, \omega_{3}, \omega_{4}, \omega, \omega}^{\bar{d} c \bar{c} d d \bar{\Lambda}}{\partial_{\omega}}_{\omega}^{d},
\end{aligned}
$$

require knowledge about six-point vertices, for which an exact consideration is numerically out of reach (similar to $\left.\Gamma^{(6)}\right)$. The lowest-order diagrams of $\Phi^{\bar{d} \bar{c} d c d \bar{d}}$ and $\Phi^{\bar{d} c \bar{c} d d \bar{d}}$ are depicted in Fig. 7(b). The simplest way of generating a non-perturbative flow is to replace bare vertices with interacting four-point vertices, which are then part of the flow. As opposed to previous proposals, namely to replace the bare interaction $U_{\alpha_{1} \alpha_{2} \alpha_{3} \alpha_{4}}$ by $\Phi_{\alpha_{1} \alpha_{2} \alpha_{3} \alpha_{4}}^{(4)}$ [26] or by an average over $\Phi^{(4)}$ with different index permutations [25], we suggest that the diagrammatically most sensible choice is the 1PI four-point vertex. Here, this amounts to replacing $-U$ by $\Gamma^{\bar{d} c \bar{c} d}=\Phi^{\bar{d} \bar{c} d c}+\Phi^{\bar{d} c \bar{c} d}-R$ [cf. Eq. (17a)]. The 1PI four-point vertex $\Gamma^{\bar{d} c \bar{c} d}$ incorporates all possible diagrams; since both $2 \mathrm{PI}$ vertices contain the totally irreducible vertex $R$, it must be subtracted. $\Gamma^{\bar{d} c \bar{c} d}$ also has the full crossing (index-permutation) symmetry as the bare interaction. Overcounting does not occur since both vertices are separated by an open $d$ line and connecting $\partial_{\Lambda} G$ to this approximation of $\Phi^{\bar{d} \bar{c} d c d \bar{d}}$ and $\Phi^{\bar{d} c \bar{c} d d \bar{d}}$ induces diagrams reducible in antiparallel and parallel lines, respectively. Since no further totally irreducible diagram for the 2PI vertices on top of the initial condition will be generated, it is consistent to use $R=-U$ in the relation for $\Gamma^{\bar{d} c \bar{c} d}$ [Eq. (17a)].

It is possible to evolve $I_{p}$ and $I_{a}$ separately, using the above described approximations in Eq. (25), and check the consistence with the parquet equations (17), interrelating both of them, during the flow. However, in the ultimately interesting combination [cf. Eq. (16) and (17)], one has the flow $\partial_{\Lambda} \Gamma^{\bar{d} c \bar{c} d}=\partial_{\Lambda} I_{p}+\partial_{\Lambda} I_{a}$. Combining the diagrams of Fig. 7(b) with full vertices and attaching the scale-derived propagator (here, equal to the singlescale propagator), we find exactly the same flow equation for the four-point vertex as given in the truncated 1PI system [Fig. 7(a)]. The replacement of $S_{\Lambda}$ by $\partial_{\Lambda} G$ in the flow of the four-point vertex when neglecting the six-point vertex, which is very natural in the above prescription, is a well known correction [19] that has been found to lead to smaller errors in Ward identities [27]. Finally, we conclude that the above simple 2PI fRG flow does not enrich the possibilities for an fRG treatment of the Fermi-edge singularity compared to the 1PI framework.

\section{CORRELATOR FROM EVOLVED VERTICES}

In this section, we start to present the results of our fRG treatment of the X-ray-edge singularity. First, we perform the fRG flow of vertices and construct the particle-hole susceptibility at the end of the flow. More precisely, we
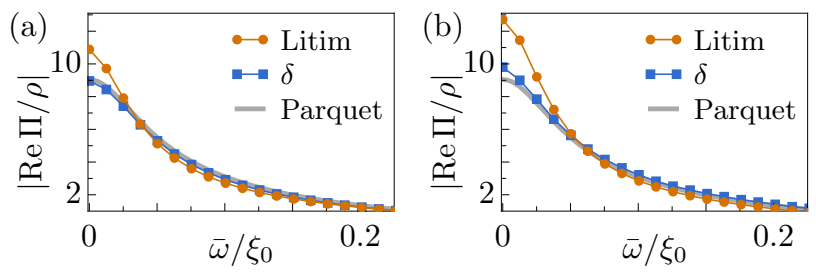

FIG. 8. (Color online) (a) Particle-hole susceptibility $\Pi$ computed via $\Gamma^{(4)}$ [Eq. (16)], which is obtained from a numerical solution of the truncated flow [cf. Fig. 7(a)]. Different results are generated using a Litim or $\delta$ regulator [cf. Eqs. (18), (19)] and compared to the leading log formula (14). (b) $\Pi$ obtained from a numerical solution of the flow in the light-matter system [Figs. 7(a), 12]. Stronger deviations (for both regulators) from the parquet curve compared to (a) occur since the truncated photon flow neglects derivatives of parallel bubbles.

examine the flow equation (22) in more detail and compare the resulting form of the particle-hole susceptibility, obtained from the relation (16), with the leading log result (14). We briefly check whether it is useful to perform a (multi-channel) Hubbard-Stratonovich transformation to generate parquet diagrams in the particle-hole susceptibility from combining several 1PI vertices, finding that this is not the case.

\section{A. Fermionic four-point vertex}

According to Eq. (16), the fermionic four-point vertex is sufficient to compute the particle-hole susceptibility. In Eq. (22), we have already given its flow equation. Since a vertex with more than four arguments (and a meaningful resolution in frequency space) is numerically intractable, we neglect the six-point vertex by truncation and obtain the simplified flow for $\Gamma^{(4)}$ illustrated in Fig. 7(a).

Solving this flow equation numerically with the initial condition $\Gamma_{\Lambda_{i}}^{(4)}=-U$, the final form of the particle-hole susceptibility [using Eq. (16)] is shown in Fig. 8(a). We find overall qualitative agreement between both the numerical and the analytic curve. Quantitatively, there are disagreements to the leading log result depending on the choice of regulator, which originate from neglecting $\Gamma^{(6)}$ in the flow of Fig. 7(a). The reason why the $\delta$ regulator yields much better results than the Litim regulator has recently been clarified in Ref. 21: The former gives less weight to multiloop corrections that are neglected in the present approach.

Let us briefly indicate which types of differentiated diagrams are missing in the flow equation when neglecting $\Gamma^{(6)}$ : One can easily check, by inserting the second-order diagrams of $\Gamma^{(4)}$ (cf. Fig. 5) on the l.h.s. and the bare vertex on the r.h.s., that the truncated flow equation [Fig. 7(a)] is satisfied at second order in the interaction. Note that (without fermionic self-energies) a diagram is simply differentiated by summing up all copies of this 


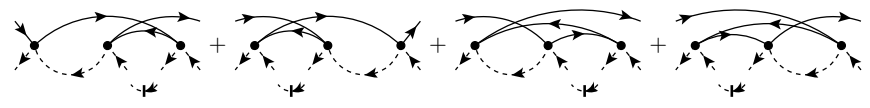

FIG. 9. Third-order contributions from the six-point vertex to the flow of $\Gamma^{(4)}$ via $\operatorname{STr}\left\{\Gamma^{(6)} S\right\}$, neglected by the truncated flow in Fig. 7(a). ( $S$ is graphically separated for clarity.)

diagram in which one $d$ line is replaced by a single-scale propagator $S=\partial_{\Lambda} G_{0}$ at any position (product rule). At third order, however, the simplified flow equation is no longer fulfilled since the six-point vertex [neglected in Fig. 7(a)] starts contributing. Indeed, the four terms coming from $\operatorname{STr}\left\{\Gamma^{(6)} S\right\}$, depicted in Fig. 9 (but neglected in the present scheme), generate the remaining derivatives of third-order parquet diagrams (cf. Fig. 5).

We emphasize that all (differentiated) diagrams generated by the truncated flow [Fig. 7(a)] are of the parquet type. Indeed, totally (two-particle-) irreducible diagrams of $\Gamma^{(4)}$ exceeding the bare vertex [corresponding to higherorder contributions of $R$ in the parquet equations (17)] require proper inclusion of the six-point vertex (and intraband four-point vertices). Similar to the recipe given in Sec. III, the truncated flow builds on the bare vertex by incorporating antiparallel and parallel bubbles and therefore only generates parquet graphs. Within the class of leading log diagrams, the six-point vertex is needed to provide all derivatives of diagrams of $\Gamma^{(4)}$, starting at third order in $U$ (cf. Fig. 9). In fact, it is easy to see that, in the fRG hierarchy, the parquet graphs comprise (1PI as well as 2PI) $n$-point vertices of arbitrarily large $n$ : Cutting a valence-band line (without leaving a single conduction-band line in the case of a 1PI description) generates a vertex of order two higher without leaving the class of parquet graphs. The corresponding higher-point vertices are required in the flow via the universal contribution $\operatorname{STr}\left\{\Gamma_{\Lambda}^{(n+2)} S_{\Lambda}\right\}$ or $\operatorname{STr}\left\{\Phi_{\Lambda}^{(n+2)} \partial_{\Lambda} G_{\Lambda}\right\}$ [cf. Eqs. (20), (23b)]. Simply truncating the (purely fermionic) fRG hierarchy of flow equations will thus always dismiss contributions to parquet graphs.

The question of how to sum up all parquet diagrams in the fermionic four-point vertex via fRG is beyond the scope of the present work and is addressed in Ref. 21 using a multiloop flow. Here, instead, we explore various other ways of computing $\Pi_{\bar{\omega}}$ by using one-loop fRG, proceeding with auxiliary bosonic fields.

\section{B. Hubbard-Stratonovich fields}

Hubbard-Stratonovich (HS) transformations are used in the context of several approximation techniques in many-body problems. Such an exact transformation reformulates the fermionic two-particle interaction in terms of propagating auxiliary particles. For instance, the lowestorder contribution to a bosonic self-energy already encodes a ladder summation in the corresponding susceptibility. For a parquet resummation, it seems therefore sensible

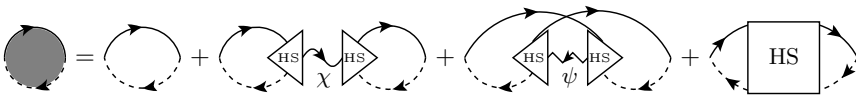

FIG. 10. Particle-hole susceptibility after a HS transformation, determined by HS three-point vertices and the four-point vertex $\Gamma_{\mathrm{HS}}^{(4)}$ (white square). Wavy and zig-zag lines denote dressed bosonic propagators. Both three-point vertices $\Gamma^{\bar{c} d \chi}$ and $\Gamma^{\bar{c}} \bar{d} \psi / i$ are depicted by a triangle and can be distinguished by the attached bosonic line.

to perform a multi-channel HS transformation [7]. With bosonic fields for the exchange $(\chi)$ and pairing $(\psi)$ channels, one has the identification

$$
\begin{aligned}
S_{\mathrm{HS}} & =\int_{\bar{\omega}} U_{\chi}^{-1} \bar{\chi}_{\bar{\omega}} \chi_{\bar{\omega}}+\frac{1}{\sqrt{\beta}} \int_{\bar{\omega} \omega}\left(\bar{c}_{\bar{\omega}+\omega} d_{\omega} \chi_{\bar{\omega}}+\bar{d}_{\omega} c_{\bar{\omega}+\omega} \bar{\chi}_{\bar{\omega}}\right) \\
& +\int_{\bar{\omega}} U_{\bar{\psi}}^{-1} \bar{\psi}_{\bar{\omega}} \psi_{\bar{\omega}}+\frac{i}{\sqrt{\beta}} \int_{\bar{\omega} \omega}\left(\bar{c}_{\bar{\omega}+\omega} \bar{d}_{\omega} \psi_{\bar{\omega}}-d_{\omega} c_{\bar{\omega}+\omega} \bar{\psi}_{\bar{\omega}}\right), \\
S_{\mathrm{int}} & =U \int_{\omega \nu \bar{\omega}}^{\prime} \bar{d}_{\omega} d_{\nu} \bar{c}_{\bar{\omega}+\nu} c_{\bar{\omega}+\omega} \rightarrow S_{\mathrm{HS}}, \quad U_{\chi}+U_{\psi}=U .
\end{aligned}
$$

Note that one can also set $U_{\chi}$ or $U_{\psi}$ to zero, such that one HS field effectively decouples from the system.

The more general relation between the particle-hole susceptibility and $1 \mathrm{PI}$ vertices in the presence of bosonic fields [cf. Eq. (6.92) of Ref. 20] is illustrated in Fig. 10. Threepoint vertices (denoted by triangles) and full bosonic propagators (wavy and zig-zag line) contribute to the correlation function. This proves beneficial in terms of computational effort as, next to the bosonic self-energies, the three-point vertices $\Gamma_{\omega, \omega-\bar{\omega}, \bar{\omega}}^{\bar{c} d \chi}$ and $\Gamma_{\omega, \bar{\omega}-\omega, \bar{\omega}}^{\bar{c} \bar{d} \psi} / i$ (with initial condition unity) contain less arguments compared to the four-point vertex. However, in Fig. 10, we see that the particle-hole susceptibility is still directly affected by the fermionic four-point vertex (which is one-particleirreducible in fermionic as well as bosonic lines). The second and third summand on the r.h.s. take the role of a four-point vertex reducible $\chi$ and $\psi$ lines, respectively, and the actual four-point vertex still covers all contributions irreducible in these lines. Although the HS transformation by construction ensures that the fourpoint vertex does not contribute to first order, it does comprise indispensable diagrams starting at second order in the interaction.

In Fig. 11(a), we show the simplest diagrams of $\Gamma_{\mathrm{HS}}^{(4)}$ after the transformation, which now start at second order in $U$. The lowest-order contributions to these diagrams, obtained by using bare bosonic propagators, represent the second-order ladder [with weight $U_{\psi}^{2}=\left(G_{0}^{\psi}\right)^{2}$ ] and secondorder crossed diagram [with weight $U_{\chi}^{2}=\left(G_{0}^{\chi}\right)^{2}$ ], known from Fig. 2 [cf. Figs. 3(b) and 5]. The main contributions of the exchange $(\chi)$ and pairing $(\psi)$ boson in Fig. 10 are reducible in the antiparallel and parallel (two-particle) channels, respectively. Correspondingly, the lowest-order diagrams of $\Gamma_{\mathrm{HS}}^{(4)}$ in Fig. 11(a) built from $\chi$ and $\psi$ lines are reducible in the complementary channels, i.e., in parallel 

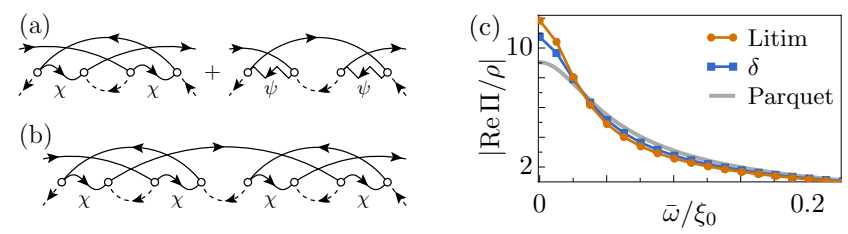

FIG. 11. (Color online) (a) After a HS transformation, $\Gamma_{\mathrm{HS}}^{(4)}$ contributes with the above diagrams, starting at second order, where white circles denote the bare three-point vertices, equal to unity. It is therefore needed to produce all parquet graphs in the correlator. (b) Whereas diagrams of the four-point vertex in (a) that are built from $\chi$ lines are irreducible in the (corresponding) antiparallel channel, starting at fourth order, diagrams with $\chi$ lines that are reducible in antiparallel (fermionic) lines occur, too. (c) Particle-hole susceptibility $\Pi$ computed via the relation in Fig. 10 without $\Gamma_{\mathrm{HS}}^{(4)}$, where bosonic self-energies and three-point vertices are obtained from the truncated fRG flow (27), (28), and the interaction strength is divided equally between both channels, $U_{\chi}=U / 2=U_{\psi}$.

and antiparallel (fermionic) lines, respectively. However, starting at fourth order in the interaction, also four-pointvertex diagrams with $\chi$ lines reducible in the antiparallel channel exist, as is demonstrated in Fig. 11(b) and analogously occurs with $\psi$ lines in the parallel channel. In fact, the diagrams in Fig. 11(a) can be used as building blocks that replace the bare interaction in the original parquet diagrams [cf. Fig. 5] to construct diagrams of $\Gamma_{\mathrm{HS}}^{(4)}$. Yet, this still covers only a fraction of the possible diagrams. We conclude that obtaining the full weight for higher-order parquet contributions to $\Pi$ via the relation in Fig. 10 requires a complicated, parquet-like resummation of diagrams containing fermionic and bosonic lines in the four-point vertex.

The flow equations for the HS self-energies and threepoint vertices can be deduced from the fundamental flow equation (20). When neglecting four-point and higher vertices, they take a form which has already been given in Eqs. (44), (45) of Ref. 7. We repeat them here for the sake of completeness and later purposes. The flow of the self-energies is given by

$$
\begin{aligned}
& \partial_{\Lambda} \Pi_{\Lambda, \bar{\omega}}^{\chi}=\int_{\omega}^{\prime} S_{\Lambda, \omega}^{d} G_{\bar{\omega}+\omega}^{c}\left(\Gamma_{\Lambda, \bar{\omega}+\omega, \omega, \bar{\omega}}^{\bar{c} d \chi}\right)^{2}, \\
& \partial_{\Lambda} \Pi_{\Lambda, \bar{\omega}}^{\psi}=\int_{\omega}^{\prime} S_{\Lambda, \omega}^{d} G_{\bar{\omega}-\omega}^{c}\left(\Gamma_{\Lambda, \bar{\omega}-\omega, \omega, \bar{\omega}}^{\bar{c} \bar{d} \psi} / i\right)^{2} .
\end{aligned}
$$

For the three-point vertices, one obtains

$$
\begin{aligned}
& \partial_{\Lambda} \Gamma_{\Lambda, \omega, \omega-\bar{\omega}, \bar{\omega}}^{\bar{c} d \chi}=\int_{\omega^{\prime}}^{\prime} S_{\Lambda, \omega^{\prime}}^{d} \Gamma_{\Lambda, \bar{\omega}+\omega^{\prime}, \omega^{\prime}, \bar{\omega}}^{\bar{c} d \chi} G_{\bar{\omega}+\omega^{\prime}}^{c} \\
& \quad \times \Gamma_{\Lambda, \bar{\omega}+\omega^{\prime}, \omega-\bar{\omega}, \omega+\omega^{\prime}}^{\bar{c} \bar{d} \psi} / i G_{\Lambda, \omega+\omega^{\prime}}^{\psi} \Gamma_{\Lambda, \omega, \omega^{\prime}, \omega+\omega^{\prime}}^{\bar{c} \bar{d} \psi} / i \\
& \partial_{\Lambda} \Gamma_{\Lambda, \omega, \bar{\omega}-\omega, \bar{\omega}}^{\bar{c} \bar{d} \psi} / i=\int_{\omega^{\prime}}^{\prime} S_{\Lambda, \omega^{\prime}}^{d} \Gamma_{\Lambda, \bar{\omega}-\omega^{\prime}, \omega^{\prime}, \bar{\omega}}^{\bar{c} \bar{d} \psi} / i G_{\bar{\omega}-\omega^{\prime}}^{c} \\
& \quad \times \Gamma_{\Lambda, \bar{\omega}-\omega^{\prime}, \bar{\omega}-\omega, \omega-\omega^{\prime}}^{\bar{c} d \chi} G_{\Lambda, \omega-\omega^{\prime}}^{\chi} \Gamma_{\Lambda, \omega, \omega^{\prime}, \omega-\omega^{\prime}}^{\bar{c} d \chi}
\end{aligned}
$$

(a)

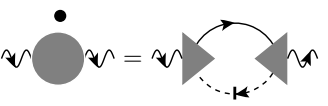

(b)

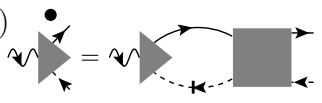

FIG. 12. Truncated flow equations for (a) the photon selfenergy $\Pi$ (depicted as circle) and (b) the photon three-point vertex $\Gamma^{\bar{c} d \gamma}$ (depicted as triangle), where the contributions of $\Gamma^{\bar{d} d \gamma \bar{\gamma}}$ [Eq. (29a)] and $\Gamma^{\bar{c} d \gamma \bar{d} d}$ [Eq. (29b)] are neglected. External (rapidly oscillating) wavy lines denote amputated photon legs. Note that the truncated flow of the four-point vertex $\Gamma^{\bar{d} c \bar{c} d}$ is still given by Fig. $7(\mathrm{a})$.

To gauge the importance of the HS four-point vertex, we have numerically solved the fRG flow in the HS-transformed system [Eqs. (27), (28)]. The resulting particle-hole susceptibility shown in Fig. 11(c), which is computed using the relation of Fig. 10 without $\Gamma_{\mathrm{HS}}^{(4)}$, shows much stronger deviations from the leading log result than Fig. 8(a), which was obtained using only $\Gamma^{(4)}$ This provides additional, numerical evidence that a HS transformation does not save us from having to calculate the fermionic four-point vertex.

\section{FLOWING SUSCEPTIBILITY}

An alternative approach to calculating the particle-hole susceptibility from renormalized 1PI vertices is based on the identification of $\Pi$ as a bosonic self-energy. In Eq. (13), we have shown how $\Pi$ is obtained from the self-energy of a rescaled photon field in the limit of its propagator (containing the dipole matrix element) going to zero. Flow equations for the photon self-energy without internal photon propagation thus describe the flow of the particle-hole susceptibility. It should be noted that this appears natural given the interpretation of polariton physics, but can also be seen as a mere computational trick in order to directly include a susceptibility in the fRG flow. In this section, we consider the flow of the photon self-energy in different levels of truncation and comment on the related publication by Lange et al. [7].

\section{A. Dynamic four-point vertex - numerical solution}

In the extended theory of the light-matter (photon and fermion) system, we derive from the fundamental flow equation (20) the flow of the photon self-energy and three-point vertex:

$$
\begin{aligned}
& \partial_{\Lambda} \Pi_{\Lambda, \bar{\omega}}= \int_{\omega}^{\prime} S_{\Lambda, \omega}^{d}\left[G_{\bar{\omega}+\omega}^{c}\left(\Gamma_{\Lambda, \bar{\omega}+\omega, \omega, \bar{\omega}}^{\bar{c} d \gamma}\right)^{2}+\Gamma_{\Lambda, \bar{\omega}, \bar{\omega}, \omega, \omega}^{\gamma \bar{\gamma} \bar{d} d}\right] \\
& \partial_{\Lambda} \Gamma_{\Lambda, \omega, \omega-\bar{\omega}, \bar{\omega}}^{\bar{c} d \gamma}=\int_{\omega^{\prime}}^{\prime} S_{\Lambda, \omega^{\prime}}^{d}\left(\Gamma_{\Lambda, \bar{\omega}+\omega^{\prime}, \omega^{\prime}, \bar{\omega}}^{\bar{c} d \gamma} G_{\bar{\omega}+\omega^{\prime}}^{c}\right. \\
& \times \Gamma_{\Lambda, \omega^{\prime}, \bar{\omega}+\omega^{\prime}, \omega, \omega-\bar{\omega}}^{\bar{d} c \bar{c} d}+\Gamma_{\Lambda, \omega, \omega-\bar{\omega}, \bar{\omega}, \omega^{\prime}, \omega^{\prime}}^{\bar{c} d \gamma \bar{d} d} .
\end{aligned}
$$


(a)

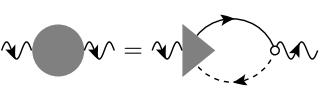

(b)

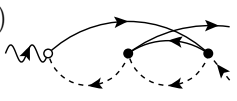

FIG. 13. (a) Schwinger-Dyson equation between photon selfenergy and three-point vertex, where the small white circle denotes a bare photon three-point vertex, equal to unity. (b) Second-order diagram of the three-point vertex, which [according to (a)] is responsible for the crossed diagram in the photon self-energy, viz., the particle-hole susceptibility (cf. Fig. 2).

The flow of $\Gamma^{\bar{d} c \bar{c} d}$, relevant for the second differential equation (29b), is still given by Eq. (22). In general, threepoint vertices connecting bosons and fermions would alter the flow of $\Gamma^{\bar{d} c \bar{c} d}$, but in the limit $G_{0}^{\gamma} \rightarrow 0$ these terms drop out. Similarly, in the absence of propagating photons, one finds that the (interband) flow of $\Gamma^{\gamma \bar{\gamma} \bar{d} d}$ is only determined by five- and six-point vertices. At our level of truncation $\Gamma_{\Lambda}^{(n>4)}=\Gamma_{\Lambda_{i}}^{(n>4)}=0$, it is therefore consistent to set $\Gamma_{\Lambda}^{\gamma \bar{\gamma} \bar{d} d}=\Gamma_{\Lambda_{i}}^{\gamma \bar{\gamma} \bar{d} d}=0$ alongside $\Gamma_{\Lambda}^{\bar{c} d \gamma \bar{d} d}=\Gamma_{\Lambda_{i}}^{\bar{c} d \gamma \bar{d} d}=0$. The resulting simplified flow is illustrated in Fig. 12.

Note that the diagrammatic expansion of the threepoint vertex $\Gamma^{\bar{c} d \gamma}$ is immediately deduced from the Schwinger-Dyson equation [cf., e.g., Fig. 11.6(b) of Ref. 20] shown in Fig. 13(a). As a consequence of truncation, the connection between $\Pi$ and $\Gamma^{\bar{d} c \bar{c} d}$ generated by the flow (via $\Gamma^{\bar{c} d \gamma}$, cf. Fig. 12) violates the basic relation between susceptibility and four-point vertex that was given in Eq. (16). This is, however, intended in order to obtain new resummations, given an approximate four-point vertex, from the explicit photon flow.

The numerical solution of the triple set of flow equations for $\Pi, \Gamma^{\bar{c} d \gamma}$ (Fig. 12) and $\Gamma^{\bar{d} c \bar{c} d}$ [Fig. 7(a)] results in the particle-hole susceptibility shown in Fig. 8(b). The agreement between the numerical solution and the parquet formula is worse compared to Fig. 8(a), where only $\Gamma^{(4)}$ was used to compute $\Pi$. The reason is that the additional flow equations in Fig. 12 exclusively contain antiparallel $S^{d}-G^{c}$ lines. They therefore induce an imbalance between the two-particle channels and neglect important contributions of diagrams with parallel lines. This begins with the crossed diagram at second order (cf. Fig. 2), which is known [15] to give a positive contribution to the particle-hole susceptibility and thus reduce the infrared divergence.

So far, the more complicated way to generate the particle-hole susceptibility from the four-point vertex, namely the additional photon flow [Eq. (29), Fig. 12] instead of the direct relation [Eq. (16), Fig. 3(b)], has led to worse agreement with the leading log formula. It is an underlying expectation of (vertex-expanded) fRG that, by incorporating more vertices in the flow, one improves the results, coming closer to the exact, infinite hierarchy of flow equations and having agreement with higher orders in perturbation theory. By contrast, in the next section, we show that if we approximate $\Gamma^{\bar{d} c \bar{c} d}$ in the simplest fashion possible - namely by the bare vertex - we actually reproduce the precise leading log result.

\section{B. Static four-point vertex - analytic solution}

The enormous simplification of using the bare four-point vertex throughout the flow has hardly any justification. Yet, we will show that, with this simplification, the flow equations can be solved analytically to yield the parquet result without further approximations. This demonstrates that one cannot judge about the content of the diagrammatic resummation solely based on the final result for a specific quantity. We will first present a purely algebraic derivation of the leading log formula for the particle-hole susceptibility and then illustrate the steps to diagrammatically understand the underlying structure.

Let us adopt a harsh but concise truncation of the flow equations: we keep all 1PI vertices starting from the four-point vertex at their initial value. The only (interband) contribution with a non-vanishing value at $\Lambda_{i}$ is the fermionic four-point vertex $\Gamma_{\Lambda}^{\bar{d} c \bar{c} d}$, which thus remains equal to $-U$ throughout the flow. The simplified flow equations [cf. Eq. (29)] then read

$$
\begin{aligned}
\partial_{\Lambda} \Pi_{\Lambda, \bar{\omega}} & =\int_{\omega}^{\prime} S_{\Lambda, \omega}^{d} G_{\bar{\omega}+\omega}^{c}\left(\Gamma_{\Lambda, \bar{\omega}+\omega, \omega, \bar{\omega}}^{\bar{c} d \gamma}\right)^{2} \\
\partial_{\Lambda} \Gamma_{\Lambda, \omega, \omega-\bar{\omega}, \bar{\omega}}^{\bar{c} d \gamma} & =-U \int_{\omega^{\prime}}^{\prime} S_{\Lambda, \omega^{\prime}}^{d} G_{\bar{\omega}+\omega^{\prime}}^{c} \Gamma_{\Lambda, \bar{\omega}+\omega^{\prime}, \omega^{\prime}, \bar{\omega}}^{\bar{c} d \gamma}
\end{aligned}
$$

The important observation is that the first derivative (and consequently any higher derivative) of $\Gamma_{\Lambda}^{\bar{c} d \gamma}$ is independent of $\omega$, i.e., completely independent of the first argument. (The second argument is fixed by conservation, anyway.) Since also the initial condition is independent of the first argument, the vertex only depends on $\bar{\omega}$, but not on $\omega$, for all scales. (This is a consequence of our truncation as diagrams of $\Gamma_{\Lambda}^{\bar{c} d \gamma}$ such as the one in Fig. 13(b), corresponding to the crossed diagram in the particle-hole susceptibility, do depend on the fermionic frequencies.)

Since $\Gamma_{\Lambda}^{\bar{c} d \gamma}$ is independent of $\omega$, the differential equations (30) can be dramatically simplified: Using the definition $g_{\Lambda, \bar{\omega}}=\left(\Gamma_{\Lambda, \cdot, \cdot \bar{\omega}}^{\bar{c} d \gamma}\right)^{2}$, we get

$$
\begin{aligned}
& \partial_{\Lambda} g_{\Lambda, \bar{\omega}}=-2 U g_{\Lambda, \bar{\omega}} \int_{\omega}^{\prime} S_{\Lambda, \omega}^{d} G_{\bar{\omega}+\omega}^{c}, \\
& \partial_{\Lambda} \Pi_{\Lambda, \bar{\omega}}=g_{\Lambda, \bar{\omega}} \int_{\omega}^{\prime} S_{\Lambda, \omega}^{d} G_{\bar{\omega}+\omega}^{c}=-\frac{1}{2 U} \partial_{\Lambda} g_{\Lambda, \bar{\omega}} .
\end{aligned}
$$

Evidently, $g_{\Lambda, \bar{\omega}}$ is given by an exponential of an auxiliary function $f_{\Lambda, \bar{\omega}}$,

$$
g_{\Lambda, \bar{\omega}}=g_{\Lambda_{i}, \bar{\omega}} e^{-2 u f_{\Lambda, \bar{\omega}}}, f_{\Lambda, \bar{\omega}}=\int_{\Lambda_{i}}^{\Lambda} \mathrm{d} \Lambda^{\prime} \int_{\omega}^{\prime} S_{\Lambda^{\prime}, \omega}^{d} G_{\bar{\omega}+\omega}^{c} / \rho,
$$

and the self-energy becomes

$$
\Pi_{\Lambda, \bar{\omega}}=\Pi_{\Lambda_{i}, \bar{\omega}}-\frac{g_{\Lambda_{i}, \bar{\omega}}}{2 U}\left[e^{-2 u f_{\Lambda, \bar{\omega}}}-1\right] .
$$

Inserting the boundary conditions $\Pi_{\Lambda_{i}}=0$ and $g_{\Lambda_{i}}=1$, 
(a)

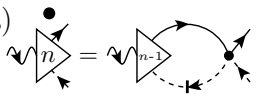

(b)

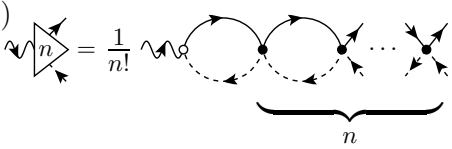

FIG. 14. (a) Flow equation for an approximate $\Gamma^{\bar{c} d \gamma}$ (at order $n)$ when $\Gamma^{\bar{d} c \bar{c} d}$ is reduced to its bare part [cf. Fig. 12(b)]. (b) Its solution, given by sequence of bubbles with a prefactor $1 / n$ !, a bare photon three-point vertex (equal to unity) and $n$ bare electronic interaction vertices.

when $\Lambda$ flows from $\infty$ to 0 , we get

$$
\Pi_{\bar{\omega}}=\frac{1}{2 U}\left[1-e^{-2 u f_{\bar{\omega}}}\right], \quad f_{\bar{\omega}}=\int_{\infty}^{0} \mathrm{~d} \Lambda \int_{\omega}^{\prime} S_{\Lambda, \omega}^{d} G_{\bar{\omega}+\omega}^{c} / \rho .
$$

So far, fermionic self-energies have not been neglected, yet. However, for the X-ray-edge singularity, we can use $S_{\Lambda}^{d}=\partial_{\Lambda} G_{\Lambda}^{d}$ and the $\Lambda$-integration becomes trivial. Using the bare bubble, computed in App., Eq. (A.3), we arrive at the remarkable conclusion that our harsh truncation directly yields the leading log result:

$$
\begin{aligned}
f_{\bar{\omega}} & =\int_{\omega}^{\prime} G_{\omega}^{d} G_{\bar{\omega}+\omega}^{c} / \rho=\ln \left(\frac{i \bar{\omega}+\xi_{d}}{-\xi_{0}}\right), \\
\Pi_{\bar{\omega}} & =\frac{\rho}{2 u}\left[1-\left(\frac{i \bar{\omega}+\xi_{d}}{-\xi_{0}}\right)^{-2 u}\right] .
\end{aligned}
$$

How is this possible? We have argued above that, in the combined, truncated system of flow equations for $\Gamma^{\bar{d} c \bar{c} d}$ and photon quantities, a large class of parquet contributions is missed by the approximate flow due to a mistreatment of parallel bubbles. We will now show diagrammatically why the parquet result could nevertheless be obtained and will find that this is only possible for the $\mathrm{X}$-ray-edge singularity.

The diagrammatic solution of the simplified flow makes extensive use of the property that ladder diagrams factorize into a sequence of (particle-hole) bubbles and that, with leading log accuracy, we can ignore fermionic selfenergies and use $S^{d}=\partial_{\Lambda} G_{0}^{d}$. If we use the bare four-point vertex in the flow of the three-point vertex [Fig. 12(b)], we obtain the flow equation shown in Fig. 14(a), which interrelates contributions to $\Gamma^{\bar{c} d \gamma}$ from subsequent orders. Due to factorization, the solution to this flow equation can be expressed diagrammatically as a three-point vertex which, at order $n$, consists of $n$ consecutive particle-hole bubbles multiplied by a prefactor $1 / n$ ! [Fig. 14(b)]. The simple ladder structure is directly related to the fact that $\Gamma_{\Lambda, \omega, \omega-\bar{\omega}, \bar{\omega}}^{\bar{c} d \gamma}$ is independent of $\omega$.

Inserting this three-point vertex in the flow equation of the photon self-energy [Fig. 12(a)], we get, at order $n$, a sequence of $n+1$ bubbles with one single-scale propagator (cf. Fig. 15). Again using factorization, this is a fraction $[1 /(n+1)]$ of the derivative of the whole ladder diagram. By computing the sum $\sum_{m=0}^{n} 1 /[m !(n-m) !]=2^{n} / n$ ! in Fig. 15, one ends up with a proportionality relation (at arbitrary order $n$ ) between the derivative of the selfenergy, $\partial_{\Lambda} \Pi^{(n)}$, and the derivative of a ladder-diagram,

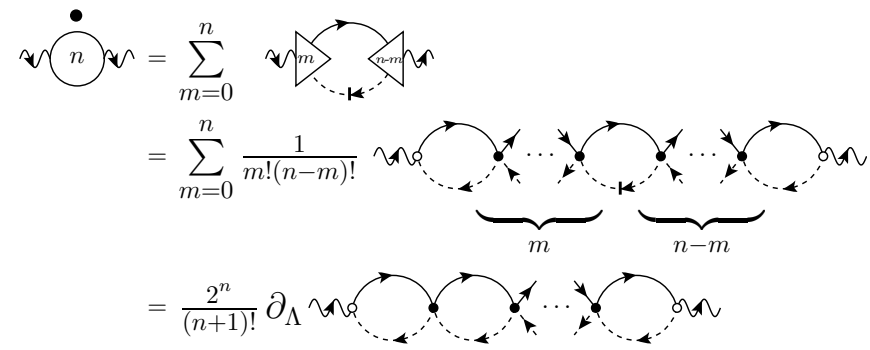

FIG. 15. Inserting the approximate $\Gamma^{\bar{c} d \gamma}$ from Fig. 14(b) in the simplified flow of $\Pi$ [Fig. 12(a)], we obtain a proportionality relation between ladder diagrams and the particle-hole susceptibility at arbitrary order $n$, in exact agreement with the leading log result [cf. Eq. (37)].

$\partial_{\Lambda} \Pi^{\mathrm{L}(n)}$. As these quantities also agree at the initial scale (both vanish when $G^{d}=0$ ), we extract an equality at all scales. Using the bare bubble as in Eq. (35a), we get

$$
\Pi_{\bar{\omega}}^{(n)}=\frac{2^{n}}{(n+1) !} \Pi_{\bar{\omega}}^{\mathrm{L}(n)}, \quad \Pi_{\bar{\omega}}^{\mathrm{L}(n)}=(-U)^{n}\left(\rho f_{\bar{\omega}}\right)^{n+1} .
$$

It remains to sum all orders $\Pi_{\bar{\omega}}^{(n)}$, i.e., sum all ladder diagrams with the appropriate prefactor [cf. Eq. (36)]. Indeed, we precisely reproduce the leading log result

$$
\begin{aligned}
\Pi_{\bar{\omega}} & =\sum_{n=0}^{\infty} \Pi_{\bar{\omega}}^{(n)}=-\frac{1}{2 U} \sum_{n=0}^{\infty} \frac{\left(-2 u f_{\bar{\omega}}\right)^{n+1}}{(n+1) !} \\
& =-\frac{\rho}{2 u}\left(e^{-2 u f_{\bar{\omega}}}-1\right)=\frac{\rho}{2 u}\left[1-\left(\frac{i \bar{\omega}+\xi_{d}}{-\xi_{0}}\right)^{-2 u}\right] .
\end{aligned}
$$

We observe that only ladder diagrams are generated by the flow while crossed diagrams do not contribute at all. However, the ladder diagrams come with prefactors, such as $1 / n$ ! in Fig. 14(b) and $2^{n} /(n+1)$ ! in Eq. (36). That the correct form of the particle-hole susceptibility is obtained at every order is then possible due to proportionality relations present in the X-ray-edge singularity, such as $\Pi^{\mathrm{L}(2)}=-3 \Pi^{\mathrm{C}(2)}$ [cf. Fig. 2], as already shown by Mahan [15] fifty years ago. Yet, these relations only hold with logarithmic accuracy, and in the more general Fermiedge singularity, where the assumption of an infinite hole mass is lifted, they hold only in a very narrow parametric regime (namely for $m / m_{h}$ being exponentially small in the coupling $u$ ) $[11,13]$. For other problems, surely such relations will only hold, if at all, subject to further assumptions. We therefore conclude that obtaining the exact first-order parquet result from a truncated fRG flow with a static four-point vertex is only possible due to a fortuitous partial cancellation of diagrams, specific to the X-ray-edge singularity.

\section{Comparison to a work by Lange et al.}

In a recent publication, Lange, Drukier, Sharma, and Kopietz [7] (LDSK) have addressed the question of using 


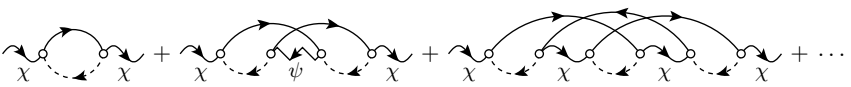

FIG. 16. Diagrams for the $\chi$ self-energy $\Pi^{\chi}$, expressed with bare three-point vertices (small white circles), equal to unity. At zeroth order in $U, \Pi^{\chi}$ is given by a bare particle-hole bubble; the only first-order contribution arises from the second diagram using $G_{0}^{\psi}=-U_{\psi}$. Starting at second order in the interaction, $\Pi^{\chi}$ contains diagrams with internal $\chi$ lines, as in the third diagram above.

fRG to tackle the X-ray-edge singularity. In fact, it is their paper which has drawn our attention to the problem at hand and deeply inspired our approach. LDSK, too, obtain the (first-order) parquet formula for the particlehole susceptibility [our Eq. (14) and their Eq. (54)] and from this draw conclusions about the relation between parquet summations and fRG. We hope that our analysis has further elucidated the derivation of the analytic result and added valuable arguments to the discussion about fRG and parquet graphs. Let us comment on some interesting points from LDSK's treatment in detail.

LDSK extract the particle-hole susceptibility from a bosonic self-energy $\left(\Pi^{\chi}\right)$ arising from a multi-channel Hubbard-Stratonovich (HS) transformation in the exchange $\left(\chi, U_{\chi}\right)$ and pairing $\left(\psi, U_{\psi}\right)$ channel. They choose (i) equal weights in both channels, $U_{\chi}=U_{\psi}$, while we will argue that only the choice $U_{\chi}=0$ allows the particle-hole susceptibility to be extracted correctly from the $\chi$ selfenergy. We will (ii) further show that, with the choice $U_{\chi}=0$, one can avoid one of the approximations made by LDSK, namely to take $u \ln \left(\xi_{0} /|\bar{\omega}|\right) \ll 1$. We will (iii) comment on the similarity between our approximate flow in the light-matter system and LDSK's flow in the HStransformed system and demonstrate numerically that including the HS-bosonic self-energies weakens the agreement with the parquet result. Furthermore, LDSK use an approximation scheme where all frequency dependencies are initially neglected and finally restored by stopping the $\mathrm{RG}$ flow at a final value of $\Lambda_{f}=\bar{\omega}$. We will (iv) give an argument, using the $\delta$ regulator, for why this scheme successfully leads to the parquet result.

(i) From the actions in Eqs. (12) and (26), it is clear that the HS field in the exchange channel, $\chi$, couples similarly to fermions as the photon field $\gamma$. However, just as for the photon [cf. Eq. (13)], it is crucial that the particle-hole susceptibility $\Pi$ be fully represented by only the leading part of the $\chi$ self-energy $\Pi^{\chi}$, i.e., the part without internal $\chi$ propagation. This is easily seen in terms of diagrams (Fig. 16): $\Pi^{\chi}$ at zeroth order is given by a conductionvalence-band particle-hole bubble, representing the zerothorder contribution to $\Pi$. At first order in the interaction, $\Pi^{\chi}$ is affected solely by $\psi$ propagation, for an intermediate $\chi$ line would result in a reducible diagram. Hence, for $\Pi^{\chi}$ to fully account for the first-order ladder diagram of $\Pi$, the bare $\psi$ propagator must have full weight, $U_{\psi}=U$. On the other hand, at second and higher orders, $\Pi^{\chi}$ contains irreducible diagrams with internal $\chi$ lines. If one chose
$U_{\chi}>0$, one would overcount these contributions and not properly generate the second-order order contribution to $\Pi$. Hence, the exact parquet graphs for $\Pi$ can only be reproduced from $\Pi^{\chi}$ by using $U_{\psi}=U$ and $U_{\chi}=0$.

(ii) Interestingly enough, with the latter choice, the approximate analytic approach of LDSK can be simplified. LDSK use $U_{\chi}=U_{\psi}=U$ and arrive at an integration of the frequency-independent, squared $\chi$ three-point vertex $g_{l}$ from a logarithmic scale parameter $l=0$ up to $l^{*}=$ $\ln \left(\xi_{0} /|\bar{\omega}|\right)$. There, they approximate $\cosh (2 u l)$ by unity [their Eq. (52)], although $u l \ll 1$ holds no longer when $l$ reaches the upper integration limit, since in the firstorder parquet regime $u l^{*}=u \ln \left(\xi_{0} /|\bar{\omega}|\right) \lesssim 1$. If one avoids this approximation and instead uses the actual $g_{l}=e^{2 u l} / \cosh (2 u l)$ for the integral in LDSK's Eq. (52), one obtains

$$
\begin{aligned}
\Pi_{\bar{\omega}}^{\chi} & =-\rho \int_{0}^{l^{*}} \mathrm{~d} l \frac{e^{2 u l}}{\cosh (2 u l)}=-\frac{\rho}{2 u} \ln \left(\frac{e^{4 u l^{*}}+1}{2}\right) \\
& =-\rho l^{*}-\rho u l^{* 2}+\mathcal{O}\left(u^{3}\right),
\end{aligned}
$$

This contains no second-order term and thus deviates already at second order in $U$ from the parquet result (14). Note that, with $\xi_{d}=0$ (as chosen by LDSK), one can only obtain the real part of the particle-hole susceptibility, solely depending on $|\bar{\omega}|$ (cf. App.). In this case, an expansion of Eq. (14) yields

$$
\begin{aligned}
\left.\operatorname{Re} \Pi_{\bar{\omega}}\right|_{\xi_{d}=0} & =\frac{\rho}{2 u}\left[1-\left(\frac{|\bar{\omega}|}{\xi_{0}}\right)^{-2 u}\right]=\frac{\rho}{2 u}\left(1-e^{2 u l^{*}}\right) \\
& =-\rho l^{*}-\rho u l^{* 2}-\frac{2}{3} \rho u^{2} l^{* 3}+\mathcal{O}\left(u^{3}\right) .
\end{aligned}
$$

The reason why performing the integral more accurately leads to an incorrect result is that the expression $g_{l}=$ $e^{2 u l} / \cosh (2 u l)$ is inaccurate at second order, since it was obtained using $U_{\chi} \neq 0$. (Consequently, $\Pi^{\chi}$ deviates from $\Pi$ starting at second order, consistent with our diagrammatic argument above.) If, instead, one uses $U_{\chi}=0$ and $U_{\psi}=U$, then Eq. (49a) of LDSK naturally yields $g_{l}=e^{2 u l}$ instead of $g_{l}=e^{2 u l} / \cosh (2 u l)$, so that the integration in their Eq. (52) reads

$$
\Pi_{\bar{\omega}}^{\chi}=-\rho \int_{0}^{l^{*}} \mathrm{~d} l e^{2 u l}=\frac{\rho}{2 u}\left(1-e^{2 u l^{*}}\right)
$$

and precisely reproduces the result of Eq. (39).

(iii) If one sets $U_{\chi}=0$ in LDSK's flow equations (44), (45) [our Eqs. (27), (28)], the three-point vertex $\Gamma^{\bar{c} \bar{d} \psi} / i$ remains equal to unity, since $G^{\chi}=0$ implies $\partial_{\Lambda} \Gamma^{\bar{c} \bar{d} \psi}=0$. If one further omits bosonic self-energy reinsertions (as done by LDSK), one has $G^{\psi}=-U_{\psi}=-U$. Hence, the resulting flow equations for $\Pi^{\chi}$ and $\Gamma^{\bar{c} d \chi}$ reduce to exactly the form of our Eq. (30) (replacing $\gamma$ by $\chi$ ). As we have shown, this flow yields the leading log result for the particle-hole susceptibility without further approximations. Actual effects of the multi-channel HS transformation become noticeable only if one actually includes bosonic self-energies 

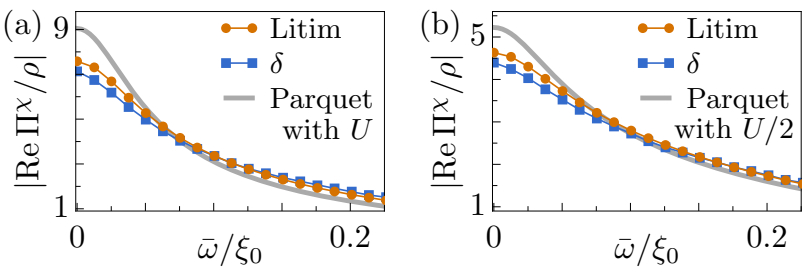

FIG. 17. (Color online) Self-energy of $\chi, \Pi^{\chi}$, as obtained from the flow in the HS-transformed system (neglecting $\Gamma^{(n>3)}$ ) [cf. Eqs. (27), (28)]. The interaction strength is divided according to (a) $U_{\chi}=0, U_{\psi}=U$ and (b) $U_{\chi}=U / 2=U_{\psi}$. Since $\chi$ propagation affects $\Pi^{\chi}$ only starting at second order (cf. Fig. 16), the result in (b) is more similar to the leading $\log$ formula with $U / 2$.

on the r.h.s. of the HS flow [Eqs. (27), (28)]. Figure 17 shows (a) that, in the case of $U_{\chi}=0, U_{\psi}=U$, this spoils the agreement with the leading $\log$ result and (b) the strikingly different outcome when using $U_{\chi}=U_{\psi}=U / 2$. In the latter case, $\Gamma^{\bar{c} \bar{d} \psi}$ contributes non-trivially, and the result is more similar to that of the leading log formula with $U / 2$, since the effect of using $U_{\chi}>0$ enters only at second and higher orders (cf. Fig. 16). We conclude that a (multi-channel) HS transformation has no advantage over the version advocated in Sec. VI of this work, based on a flowing susceptibility in the fermionic system.

(iv) In their analytic solution of the flow, LDSK use an approximation scheme where frequency dependencies in all 1PI vertices were omitted initially. Viewing this as a low-energy approximation, they let $\Lambda$ flow from $\xi_{0}$ to $\bar{\omega}$ instead of the expected range $\infty$ to 0 . From another perspective, this integration range for $\Lambda$ can be obtained by computing the "single-scale" bubble [Eq. (41)] with the $\delta$ regulator. As explained above, LDSK's system of flow equations with $U_{\chi}=0$ and $G^{\psi}=-U$ can be directly related to our photon flow in Eq. (30). We have shown that the $\bar{\omega}$-dependence enters only in the (integrated) single-scale bubble $\left[f_{\bar{\omega}}\right.$ in Eq. (34)], which can also be integrated first w.r.t. frequency and then w.r.t. $\Lambda$. Making use of the $\delta$ regulator, $\xi_{d}=0$ (such that $|\bar{\omega}| \ll \xi_{0}$ ), and the (simplified) local $c$ propagator [Eq. (11)], one readily obtains

$$
\begin{aligned}
\int_{\omega}^{\prime} S_{\Lambda, \omega}^{d} G_{\bar{\omega}+\omega}^{c} / \rho & =\int_{-\xi_{0}-\bar{\omega}}^{\xi_{0}-\bar{\omega}} \mathrm{d} \omega \operatorname{sgn}(\bar{\omega}+\omega) \frac{\delta(|\omega|-\Lambda)}{2 \omega} \\
& \approx \int_{-\xi_{0}}^{\xi_{0}} \mathrm{~d} \omega \operatorname{sgn}(\bar{\omega}+\omega) \frac{\delta(|\omega|-\Lambda)}{2 \omega} \\
& =\Theta\left(\xi_{0}-\Lambda\right) \sum_{\omega= \pm \Lambda} \frac{\operatorname{sgn}(\bar{\omega}+\omega)}{2 \omega} \\
& =\frac{\Theta\left(\xi_{0}-\Lambda\right) \Theta(\Lambda-|\bar{\omega}|)}{\Lambda} .
\end{aligned}
$$

Using this as a factor in the relevant flow equations, similarly as in Eq. (31), naturally restricts the integration range for $\Lambda$ precisely in the way chosen by LDSK.

\section{CONCLUSIONS}

We have analyzed the X-ray-edge (zero-dimensional Fermi-edge) singularity - an instructive fermionic problem with simplified diagrammatics focused on two-particle quantities, an analytic parquet and exact one-body solution. Our goal was to use the functional renormalization group to achieve a partial resummation of diagrams, to be compared to the (first-order) solution of the parquet equations. We compared results for the particle-hole susceptibility with the leading log formula in terms of Matsubara frequencies and examined the diagrammatic structure of the flow equations. We found that different realizations of a truncated, one-loop fRG flow do not fully generate the leading log diagrams.

Focusing on the flow of the fermionic four-point vertex $\Gamma^{(4)}$ first, we argued that, in the fRG hierarchy, the parquet diagrams comprise (1PI and $2 \mathrm{PI}$ ) vertices of any order, and that these higher-order vertices, obtained by cutting appropriate scale-dependent lines, universally contribute to the flow. Hence, simply truncating the fRG hierarchy of flow equations will always miss contributions to parquet graphs. We further showed that a (multichannel) Hubbard-Stratonovich transformation does not remedy this problem: Although the transformation ensures that $\Gamma_{\mathrm{HS}}^{(4)}$ does not contribute to the particle-hole susceptibility $\Pi$ at first order, it does contribute important, parquet diagrams to $\Pi$ starting at second order in the interaction, which are lost when the four-point vertex is neglected.

As a different approach, we included $\Pi$ in the fRG flow as a (leading contribution to a) photon self-energy (i.e., as a flowing susceptibility). We showed that the relation between $\Gamma^{(4)}$ and $\Pi$ generated by truncated flow equations systematically misses contributions from parallel bubbles. However, in contrast to the underlying philosophy of fRG, we found an improved result for $\Pi$ when treating the four-point vertex less accurately. In fact, we analytically reproduced the leading log formula using a truncated fRG flow that keeps four-point and higher vertices constant. We showed that, in this way, one effectively only sums up ladder diagrams, but with a set of prefactors that fortuitously turns out to precisely yield the correct form of $\Pi$. This is possible thanks to proportionality relations of ladder and crossed diagrams, which, however, only hold with logarithmic accuracy and are violated when extending the theory, e.g., to a finite-mass valence-band description. Our derivation of the (first-order) parquet result from a truncated fRG flow using a static fourpoint vertex is thus only possible due to a fortuitous partial cancellation of diagrams specific to the X-ray-edge singularity.

In related publications [21, 22], we show how the truncated flow equations can actually be extended to capture all parquet graphs. This multiloop fRG flow simulates the effect of the six-point vertex on parquet contributions and iteratively completes the derivative of diagrams in the flow equations of both four-point vertex and self-energy. 


\section{ACKNOWLEDGMENTS}

We thank D. Pimenov, D. Schimmel, and L. Weidinger for useful discussions and P. Kopietz for a helpful correspondence. We acknowledge support by the Cluster of Excellence Nanosystems Initiative Munich; F.B.K. acknowledges funding from the research school IMPRSQST.

\section{Appendix: Particle-hole bubble}

In this section, we explicitly compute the bare (interband) particle-hole bubble, needed in Sec. VI, Eq. (35). We also show that this bubble is discontinous w.r.t. the bandgap $-\xi_{d}$ at $\xi_{d}=0$. Thus, we choose $\xi_{d}$ suitably small (cf. Sec. III) but nonzero in our numerical calculations.

The bare bubble is given by the integral

$$
\begin{aligned}
\Pi_{0, \bar{\omega}} & =\int_{\omega}^{\prime} G_{0}^{c}(\bar{\omega}+\omega) G_{0}^{d}(\omega) \\
& =-i \pi \rho \int_{\omega}^{\prime} \frac{\operatorname{sgn}(\bar{\omega}+\omega) \Theta\left(\xi_{0}-|\bar{\omega}+\omega|\right)}{i \omega-\xi_{d}} \\
& =\Pi_{0,-\bar{\omega}}^{*},
\end{aligned}
$$

which we divide into three parts: $\Pi_{0, \bar{\omega}}=I_{1}+I_{2}+I_{3}$. We first consider $\bar{\omega}>0$, revert to frequency integrals in the zero-temperature limit [cf. Eq. (10)], and obtain

$$
\begin{aligned}
& I_{1}=\frac{\rho}{2 i} \int_{\bar{\omega}}^{\xi_{0}-\bar{\omega}} \frac{\mathrm{d} \omega}{i \omega-\xi_{d}}=\frac{\rho}{2} \ln \left(\frac{i \bar{\omega}-\xi_{d}}{i \xi_{0}-i \bar{\omega}-\xi_{d}}\right) \\
& I_{2}=\frac{\rho}{2 i} \int_{-\bar{\omega}}^{\bar{\omega}} \frac{\mathrm{d} \omega}{i \omega-\xi_{d}}=\frac{\rho}{2} \ln \left(\frac{-i \bar{\omega}-\xi_{d}}{i \bar{\omega}-\xi_{d}}\right) \\
& I_{2}=i \rho \int_{0}^{\bar{\omega}} \mathrm{d} \omega \frac{\xi_{d}}{\left(\xi_{d}\right)^{2}+(\bar{\omega})^{2}}=i \rho \arctan \left(\frac{\bar{\omega}}{\xi_{d}}\right) \\
& I_{3}=\frac{i \rho}{2} \int_{-\xi_{0}-\bar{\omega}}^{-\bar{\omega}} \frac{\mathrm{d} \omega}{i \omega-\xi_{d}}=\frac{\rho}{2} \ln \left(\frac{i \bar{\omega}+\xi_{d}}{i \xi_{0}+i \bar{\omega}+\xi_{d}}\right) .
\end{aligned}
$$

In the form of Eq. (A.2c), one can directly see that the integral $I_{2}$ is discontinuous w.r.t. $\xi_{d}$ at $\xi_{d}=0$. Essentially, the contribution from $I_{2}$ is needed to produce the correct phase in the susceptibility, when summing $I_{1}, I_{2}$, and $I_{3}$. Using the fact that, upon analytic continuation to real frequencies, one has $\left|i \bar{\omega}+\xi_{d}\right| \rightarrow\left|\omega+\xi_{d}+i 0^{+}\right| \ll \xi_{0}$, we obtain the approximate form

$$
\begin{aligned}
\Pi_{0, \bar{\omega}} & =\frac{1}{2} \ln \left(\frac{i \bar{\omega}+\xi_{d}}{i \bar{\omega}+\xi_{d}-i \xi_{0}}\right)+\frac{1}{2} \ln \left(\frac{i \bar{\omega}+\xi_{d}}{i \bar{\omega}+\xi_{d}+i \xi_{0}}\right) \\
& \approx \frac{1}{2} \ln \left(\frac{i \bar{\omega}+\xi_{d}}{-i \xi_{0}}\right)+\frac{1}{2} \ln \left(\frac{i \bar{\omega}+\xi_{d}}{i \xi_{0}}\right) \\
& =\ln \left(\frac{i \bar{\omega}+\xi_{d}}{-\xi_{0}}\right)
\end{aligned}
$$

which also holds for negative frequencies according to the symmetry relation $\Pi_{0, \bar{\omega}}=\Pi_{0,-\bar{\omega}}^{*}$.

If, instead, one sets $\xi_{d}=0$ in the first place, one in effect omits the contribution from $I_{2}$ [cf. Eq. (A.2c)]. With the approximation $|i \bar{\omega}| \ll \xi_{0}$, one then obtains from $I_{1}+I_{3}$ :

$$
\begin{aligned}
\left.\Pi_{0, \bar{\omega}}\right|_{\xi_{d}=0, \bar{\omega}>0} & =\frac{1}{2} \ln \left(\frac{i \bar{\omega}}{i \xi_{0}-i \bar{\omega}}\right)+\frac{1}{2} \ln \left(\frac{i \bar{\omega}}{i \xi_{0}+i \bar{\omega}}\right) \\
& \approx \ln \left(\frac{\bar{\omega}}{\xi_{0}}\right) .
\end{aligned}
$$

Reverting to positive and negative frequencies via Eq. (A.1) again, we finally get

$$
\left.\Pi_{0, \bar{\omega}}\right|_{\xi_{d}=0}=\ln \left(\frac{|\bar{\omega}|}{\xi_{0}}\right) .
$$

Having set $\xi_{d}=0$, one only obtains the real part of the particle-hole bubble, solely depending on $|\bar{\omega}|$. Moreover, in contrast to the real-frequency calculations of Roulet et. al [5], who focus on the real part and argue that the imaginary part can be reconstructed by Kramers-Kronig relations, this is not possible in the Matsubara framework, where one does not have such relations between Re $\Pi$ and $\operatorname{Im} \Pi$. We conclude that one should therefore refrain from setting $\xi_{d}=0$.
[1] A. Gabbay, Y. Preezant, E. Cohen, B. M. Ashkinadze, and L. N. Pfeiffer, Phys. Rev. Lett. 99, 157402 (2007).

[2] S. Smolka, W. Wuester, F. Haupt, S. Faelt, W. Wegscheider, and A. Imamoglu, Science 346, 332 (2014).

[3] M. Sidler, P. Back, O. Cotlet, A. Srivastava, T. Fink, M. Kroner, E. Demler, and A. Imamoglu, Nat. Phys. 13, 255 (2016).

[4] T. Giamarchi, Quantum Physics in One Dimension (Clarendon Press, 2004) p. 347.

[5] B. Roulet, J. Gavoret, and P. Nozières, Phys. Rev. 178, 1072 (1969).

[6] P. Nozières, J. Gavoret, and B. Roulet, Phys. Rev. 178, 1084 (1969).
[7] P. Lange, C. Drukier, A. Sharma, and P. Kopietz, J. Phys. A 48, 395001 (2015).

[8] I. Carusotto and C. Ciuti, Rev. Mod. Phys. 85, 299 (2013).

[9] N. S. Averkiev and M. M. Glazov, Phys. Rev. B 76, 045320 (2007).

[10] M. Baeten and M. Wouters, Phys. Rev. B 89, 245301 (2014); Eur. Phys. J. D 69, 243 (2015).

[11] D. Pimenov, Fermi-edge polaritons with finite hole mass, Master's thesis, Ludwig-Maximilians-Universität München (2015); D. Pimenov, J. von Delft, L. Glazman, and M. Goldstein, Phys. Rev. B 96, 155310 (2017).

[12] P. Nozières and C. T. De Dominicis, Phys. Rev. 178, 1097 (1969). 
[13] J. Gavoret, P. Nozières, B. Roulet, and M. Combescot, J. Phys. France 30, 987 (1969).

[14] G. D. Mahan, Phys. Rev. 153, 882 (1967).

[15] G. D. Mahan, Phys. Rev. 163, 612 (1967).

[16] A. A. Abrikosov, Physics 2, 5 (1965).

[17] K. Fukushima, Prog. Theor. Phys. 46, 1307 (1971).

[18] N. Bickers, in Theoretical Methods for Strongly Correlated Electrons, CRM Series in Mathematical Physics, edited by D. Sénéchal, A.-M. Tremblay, and C. Bourbonnais (Springer New York, 2004) pp. 237-296.

[19] W. Metzner, M. Salmhofer, C. Honerkamp, V. Meden, and K. Schönhammer, Rev. Mod. Phys. 84, 299 (2012).
[20] P. Kopietz, L. Bartosch, and F. Schütz, Introduction to the Functional Renormalization Group (Lecture Notes in Physics) (Springer, Berlin, 2010).

[21] F. B. Kugler and J. von Delft, Phys. Rev. Lett. 120, 057403 (2018).

[22] F. B. Kugler and J. von Delft, Phys. Rev. B 97, 035162 (2018).

[23] D. F. Litim, Phys. Rev. D 64, 105007 (2001).

[24] C. Wetterich, Phys. Lett. B 301, 90 (1993).

[25] J. F. Rentrop, S. G. Jakobs, and V. Meden, J. Phys. A 48, 145002 (2015).

[26] N. Dupuis, Eur. Phys. J. B 48, 319 (2005).

[27] A. A. Katanin, Phys. Rev. B 70, 115109 (2004). 